\title{
Non-Equilibrium Fluid Flow Around the Yucca Mountain Nuclear Waste Repository
}

John C. Reis

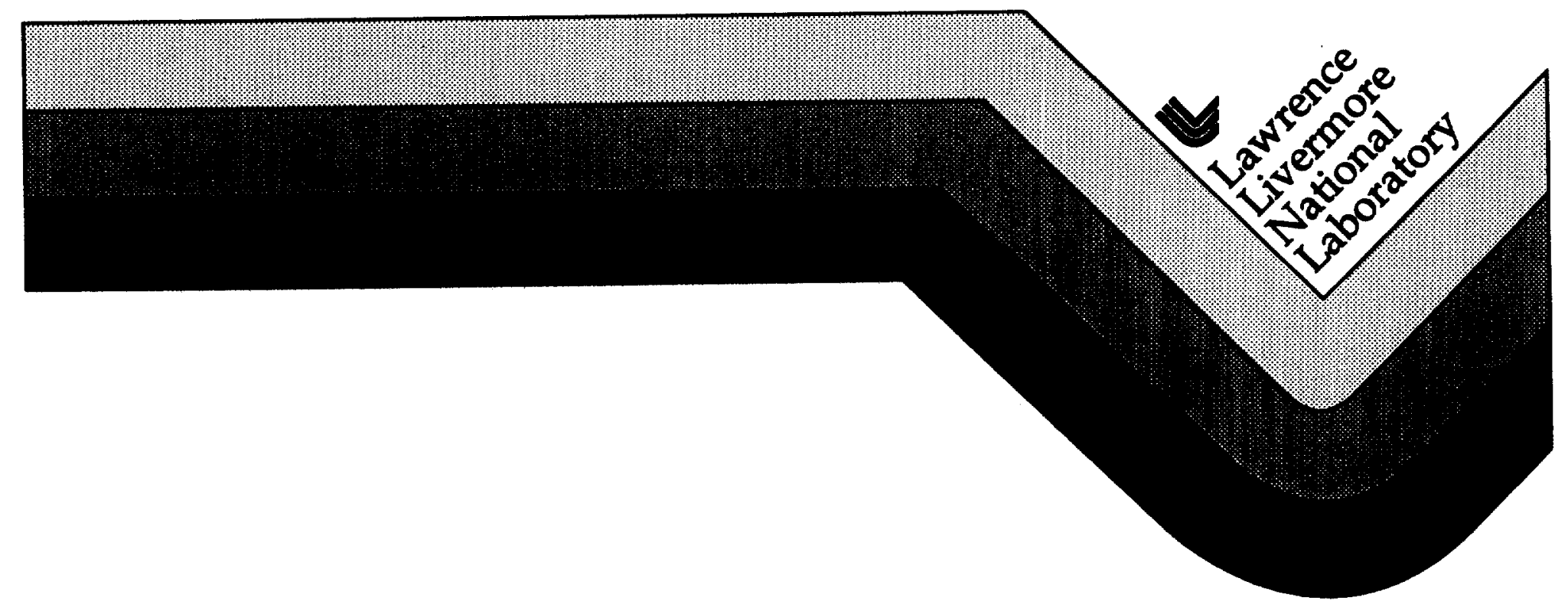




\section{DISCLAIMER}

This document was prepared as an account of work sponsored by an agency of the United States Government. Neither the United States Government nor the University of California nor any of their employees, makes any warranty, express or implied, or assumes any legal liability or responsibility for the accuracy, completeness, or usefulness of any information, apparatus, product, or process disclosed, or represents that its use would not infringe privately owned rights. Reference herein to any specific commercial product, process, or service by trade name, trademark, manufacturer, or otherwise, does not necessarily constitute or imply its endorsement, recommendation, or favoring by the United States Government or the University of California. The views and opinions of authors expressed herein do not necessarily state or reflect those of the United States Government or the University of Califomia, and shall not be used for advertising or product endorsement purposes.

Work performed under the auspices of the U.S. Department of Energy by Lawrence Livermore National Laboratory under Contract W-7405-ENG-48. 


\title{
Non-Equilibrium Fluid Flow Around the Yucca Mountain
} Nuclear Waste Repository

\author{
John C. Reis \\ Aerospace Engineering Department \\ Embry-Riddle Aeronautical University \\ Prescott, AZ 86301
}




\section{TABLE OF CONTENTS}

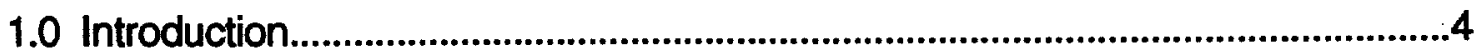

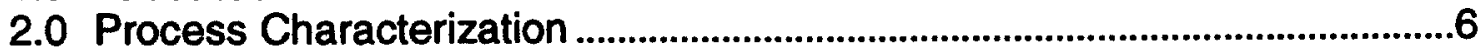

2.1 Heat Transport......................................................................................6

2.1.1 Linear Geometry .......................................................................6

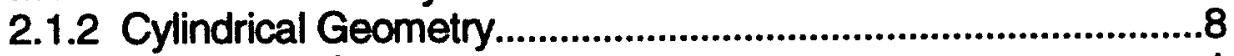

2.1.3 Spherical Geometry ..................................................................10

2.2 Fluid Transport.............................................................................................11

2.2.1 Gravity Drainage ...................................................................12

2.2.2 Counter-Current Capillary Imbibition .......................................15

2.2.3 Co-Current Capillary Imbibition ................................................16

2.2.4 Co-Current Capillary and Gravity Imbibition...........................19

2.2.5 Effect of Crossflow on Water Advance in Fractures..............22

2.2.6 Formation Pressurization From Heating..................................24

3.0 Creation of a Water Bank......................................................................................26

3.1 Height of Water Bank.................................................................................26

3.2 Vertical Gravity Drainage...........................................................................28

3.3 Gravity Drainage Around Dry Zone .........................................................30

3.4 Matrix Imbibition Rate...............................................................................34

3.5 Discussion of Water Bank.........................................................................35

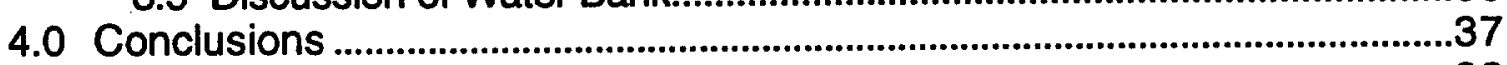

References ............................................................................................................38

Nomenclature ...............................................................................................................40 


\section{LIST OF TABLES}

Table 2.1.1 Data for Temperature Models ...................................................................42

Table 2.2.1 Data for Numerical Simulations...........................................................43

Table 2.2.2 Data for Matrix Pressurization .............................................................44

Table 3.1.1 Data for Water Bank Height..............................................................45

Table 3.2.1 Data for Gravity Drainage ........................................................................46

Table 3.3.1 Data for Merging Dry Zones ................................................................4 47

Table 3.4.1 Data for Imbibition ....................................................................................48

\section{LIST OF FIGURES}

Figure 2.1.1 Comparison of Temperature Models for Conduction.......................49

Figure 2.1.2 Boiling Front Velocities .....................................................................50

Figure 2.1.3 Relative Permeabilities for Simulation Model.....................................51

Figure 2.1.4 Capillary Pressure for Simulation Model............................................52

Figure 2.1.5 Temperature Profiles for Constant Pressure Boundary....................53

Figure 2.1.6 Temperature Profiles for No-Flow Boundary ......................................54

Figure 2.2.1 Schematic of Water Bank During Gravity Drainage ..........................55

Figure 2.2.2 Water Saturation Profiles During Gravity Drainage..........................56

Figure 2.2.3 Geometry for Counter-Current Capillary Imbibition ...........................57

Figure 2.2.4 Geometry for Co-Current Capillary Imbibition ...................................58

Figure 2.2.5 Geometry for Co-Current Capillary and Gravity Imbibition...............59

Figure 2.2.6 Schematic of Capillary Crossflow for a Layered System .................60

Figure 2.2.7 Gas Pressure Profiles................................................................................61

Figure 2.2.8 Characteristic Matrix Depressurization Time ....................................62

Figure 3.1.1 Water Saturation Distribution Ahead of Boiling Front ......................63

Figure 3.1.2 Water Bank Height in Fractures..........................................................64

Figure 3.3.1 Enthalpies for Vaporization and Conduction around Dry Zone....65

Figure 3.3.2 Geometry of Continuous Dry Zone ...................................................66

Figure 3.3.3 Ratio of Drainage Velocities Around Continuous Dry Zone...........67

Figure 3.4.1 Schematic of Experimental Apparatus.............................................68

Figure 3.4.2 Comparison of Experimental Data to Imbibition Models .................69

Figure 3.4.3 Water Bank Velocity in Fractures ..........................................................70 


\subsection{Introduction}

Yucca Mountain near Las Vegas, Nevada is being evaluated by the U.S. Department of Energy for suitability as permanent repository for the disposal of high-level radioactive wastes. A major factor in the determining its suitability is its potential to limit transport of radionuclides away from the site for tens of thousands of years. The transport mechanisms of most concem require that the waste packages be contacted by liquid water. Because the proposed repository site is above the water table, there are only a limited number of ways that liquid water can contact waste packages: storm water draining down through the formation from the surface and concentration of insitu water from thermo-hydrological processes.

After the nuclear waste has been placed in the repository, the temperature of the formation around the repository will increase from radioactive decay heating. For high thermal loads, e.g., high waste concentrations, the temperature is expected to exceed the saturation temperature of water, vaporizing the in-situ water in the pores of the surrounding formation. This will create a dry zone around individual waste packages. With further heating, the individual dry zones can merge and create a dry zone around the entire repository.

As water in the formation is vaporized, it will raise the local gas pressure. This higher pressure will drive the water vapor away from the dry zone and into the cooler formation, where it condenses. Because the liquid water is trapped in the pores from capillary forces, little if any, will be driven away from the dry zone as liquid from the increased pressure. This trapped water will boil at a rate determined by the conductive heat transfer rate through the formation. The vaporization, gas-phase transport, and subsequent condensation of the trapped water will create a steam plateau at constant temperature around the growing dry zone. The water condensing at the leading edge of the steam plateau will increase the local liquid water saturation, creating a water bank. Because this water bank will have a saturation higher than the initial water saturation, this water will be able to migrate more readily through the formation.

The movement of a water bank around the dry zone will be affected by both the pressurization of the formation around the waste packages and by gravitational forces. Water in the bank beside or below the repository will be driven downward and away from the repository by both forces and is not of particular concern. Water in the bank above the repository, however, will be driven upward by pressurization, but will be forced back downward by gravity. Under some conditions, these competing processes could create a sizable water bank above the repository.

After nuclear heating has decayed, a water bank above the repository can migrate downward by gravity drainage, rewetting the dry zone. If the formation properties were uniform and if the water bank were created symmetrically around the repository, the amount of water in the bank above the repository would be just sufficient to rewet the formation back to the repository level, leaving the formation below the repository dry.

Because of a variety of effects, however, the amount of water in the bank above the repository could be larger than that below the repository and there may be sufficient 
liquid water above the repository to rewet the formation at the repository level above its initial saturation and possibly wet the waste packages. Effects that may create an asymmetry in the water bank include thermally-induced buoyancy effects in the dry zone and the differential contribution of gravitational forces on the water bank above and below the dry zone.

Because of the importance of keeping liquid water away from the waste packages, the possible existence of a water bank above the repository raises questions about the long-term integrity of the waste packages and their ability to limit migration of radionuclides away from the repository.

Preliminary computer simulations of heat and fluid transport around the Yucca Mountain repository have suggested that a significant liquid water bank can form above the repository (Buscheck and Nitao, 1993a; Buscheck and Nitao, 1993b; Buscheck, et al., 1993; Buscheck, et al., 1994). However, a small-scale field study in the G-Tunnel at Yucca Mountain utilizing electrical heating to create a dry zone revealed no evidence of a water bank around the dry zone (Ramirez, et al., 1991).

The simulation studies predicting a water bank above the repository were based on an equilibrium continuum model (ECM) that assumed that the fractures and matrix are in capillary equilibrium. This assumption allows the fracture and matrix properties to be averaged into an equivalent medium and significantly reduces the computational time required to study repository behavior. The bulk porosity, water saturation, and permeability for ECM are given by

$$
\begin{gathered}
\phi_{b}=\phi_{f}+\left(1-\phi_{1}\right) \phi_{m}, \\
S_{b}=\frac{S_{1} \phi_{1}+S_{m}\left(1-\phi_{f}\right) \phi_{m}}{\phi_{1}+\left(1-\phi_{1}\right) \phi_{m}},
\end{gathered}
$$

and

$$
k_{b}=k_{1} \phi_{f}+k_{m}\left(1-\phi_{1}\right)
$$

respectively.

The authors of the ECM simulation studies recognized the limitations to the ECM approach to simulating fluid flow in fractured reservoirs. They reported that the actual water saturation in a bank above the repository would be lower than that calculated because non-equilibrium flow through the fractures would drain the water away from the site. Preliminary studies of fluid flow around the Yucca Mountain repository using non-equilibrium models have been reported (Nitao, 1991; Nitao and Buscheck, 1991; and Nitao et al., 1993), but these studies have not provided a quantitative evaluation of the creation of a water bank.

The objective of this study was to analytically characterize the processes that impact the creation of a water bank and to evaluate whether or not a water bank is likely to form above the repository. 


\subsection{Process Characterization}

In this section, the fundamental processes of heat and fluid transport around the proposed Yucca Mountain repository are characterized. This characterization includes developing analytical equations that model heat and fluid transport around the site.

\subsection{Heat Transport}

The radioactive decay of the nuclear wastes will act as a heat source in the repository. For a dense packing of wastes, the decay heat will raise the temperature of the repository above the in-situ boiling point of water, vaporizing any water trapped in the formation pores. This will dry the formation around the repository.

In this section, analytical expressions for temperature profiles and velocities of the boiling front at the edge of the dry zone are developed for idealized geometries. The geometries considered are linear conduction into a semi-infinite medium, radial conduction into a semi-infinite medium internally bounded by an infinitely-long cylinder, and radial conduction into a semi-infinite medium internally bounded by a sphere. In all geometries, a constant heat flux at the medium interface is assumed.

\subsubsection{Linear Geometry}

For one-dimensional conduction into a semi-infinite slab with a constant heat flux at the surface, the temperature profile is given by (Carslaw and Jaeger, 1959, pg. 75):

$$
\mathrm{T}(\mathrm{x}, \mathrm{t})=\mathrm{T}_{0}+\frac{2 \mathrm{~F}_{0}}{\lambda}\left[\sqrt{\frac{\alpha \mathrm{t}}{\pi}} \exp \left\{-\left(\frac{\mathrm{x}}{2 \sqrt{\alpha \mathrm{t}}}\right)^{2}\right\}-\frac{\mathrm{x}}{2} \operatorname{erfc}\left(\frac{\mathrm{x}}{2 \sqrt{\alpha \mathrm{t}}}\right)\right],
$$

where $T_{0}$ is the initial formation temperature, $F_{0}$ is the heat flux into the formation, $\lambda$ is the formation thermal conductivity, $\alpha$ is the formation diffusivity, $t$ is the time since the start of heating, and $x$ is the distance into the formation from the heat source.

The temperature of the formation at the heat source is given by

$$
T(0, t)=T_{0}+\frac{2 F_{0}}{\lambda} \sqrt{\frac{\alpha t}{\pi}}
$$

Over a wide range of conditions, Eq. 2.1.1.1 can be approximated by

$$
T(x, t)=T_{0}+\frac{2 F_{0}}{\lambda} \sqrt{\frac{\alpha t}{\pi}} \exp \left\{-\frac{x}{\sqrt{\alpha t}}\right\} \text {. }
$$


The "exact" equation for the temperature given by Eq. 2.1.1.1 is compared to the model given by Eq. 2.1.1.3 in Fig. 2.1.1 for the conditions given in Table 2.1.1. The model reasonably matches the exact equation except at large distances.

Solving Eq. 2.1.1.3 for $x$ yields the position at which any temperature can be found at any time:

$$
x=\sqrt{\alpha t} \ln \left[\sqrt{\frac{\alpha t}{\pi}} \frac{2 F_{0}}{\lambda\left(T(x, t)-T_{0}\right)}\right] .
$$

The position of the boiling front (edge of the dry zone) can be approximated by substituting the boiling temperature into Eq. 2.1.1.4:

$$
x_{b}=\sqrt{\alpha t} \ln \left[\sqrt{\frac{\alpha t}{\pi}} \frac{2 F_{0}}{\lambda\left(T_{b}-T_{0}\right)}\right] .
$$

The velocity of the boiling front as it moves away from the heat source is given by

$$
u_{b}=\frac{d x_{b}}{d t}=\frac{1}{2} \sqrt{\frac{\alpha}{t}}\left[1+\ln \left(\sqrt{\frac{\alpha t}{\pi}} \frac{2 F_{0}}{\lambda\left(T_{b}-T_{0}\right)}\right)\right] .
$$

This velocity is plotted as a function of time in Fig. 2.1.2 using the data in Table 2.1.1. The maximum velocity of the boiling from for these conditions is on the order of one meter per year.

The above analysis assumes that vaporization and condensation do not significantly affect the conductive temperature profile and the velocity of the boiling front. This assumption was numerically evaluated using the NUFT code developed by Nitao $(1993,1994)$.

A one-dimensional array of 100 grid blocks was used, with a grid block size of 1 cubic meter. The heat source was placed in the center grid block. The grid was arranged vertically to allow the effects of gravity to be simulated. The absolute permeability was one darcy. The relative permeabilities used were taken from Pirson, et al. (1964). The water and gas relative permeabilities are provided in Fig. 2.1.3. The capillary pressure data, adapted from Brown (1951) are shown in Fig. 2.1.4. Other data are given in Table 2.1.2. Two boundary conditions were used for the pressure at the top grid. The first boundary condition was a constant pressure to simulate the atmosphere. The second boundary condition was a no-flow boundary. In all cases, a no-flow boundary condition was imposed at the bottom grid.

The simulated temperature profiles after 10 years are shown in Fig. 2.1.5 for the constant pressure upper boundary, with and without the effects of gravity. For comparison, the theoretical temperature for simple conduction given by Eq. 2.1.1.1 is also shown. When vaporization is present, there is a depression in the peak temperature at the heat source as some of the sensible heat is converted to latent 
heat. For the case of no gravity, a small steam plateau was located above the heat source and no plateau was located below. For the case of gravity being present, a very large steam plateau was above the heat source and a small one below. The steam plateaus above the heat source were created by the upward migration of steam vapor as it was generated at the boiling front. This upward migration was the result of the constant pressure boundary condition at the top of the model allowing flow out of the model to relieve the formation pressure.

The simulated temperature profiles for the no-flow boundary condition are shown in Figure 2.1.6. In this case, the temperature profile for no gravity is symmetric about the heat source with well-defined steam plateaus. The temperature of the plateaus, however, is elevated above $100^{\circ} \mathrm{C}$ because the no-flow boundary allowed the system to pressurize and elevate the saturated temperature.

Figures 2.1.5 and 2.1.6 show that the simulated position of the boiling front is within about a factor of two of that predicted by the analytical theory for conduction heat transfer. This agreement is believed to be sufficiently accurate to justify the use of the theoretical temperature profiles to estimate the correct order of magnitude of the expansion of the dry zone, even when vaporization and condensation occur.

\subsubsection{Cylindrical Geometry}

For one-dimensional radial conduction into a medium bounded internally by a circular cylinder with a constant heat flux at its surface, the radial temperature profile at large times can be approximated by (Carslaw and Jaeger, 1959, pg. 341):

$$
T(r, t)=T_{0}+\frac{R F_{0}}{2 \lambda}\left\{\left(1+\frac{R^{2}}{2 \alpha t}\right) \ln \left(\frac{4 \alpha t}{C r^{2}}\right)+\frac{1}{4 \alpha t}\left[R^{2}+r^{2}-2 R^{2} \ln \left(\frac{R}{r}\right)\right]\right\},
$$

where $T_{0}$ is the initial formation temperature, $F_{0}$ is the heat flux into the formation, $\lambda$ is the formation thermal conductivity, $\alpha$ is the formation diffusivity, $t$ is the time since the start of heating, $R$ is the internal radius of the cylinder providing the heat flux, $C$ is constant equal to 1.781 , and $r$ is the distance into the formation from the edge of the cylinder.

The temperature of the formation at the heat source is given by

$$
T(0, t)=T_{0}+\frac{R F_{0}}{2 \lambda}\left\{\left(1+\frac{R^{2}}{2 \alpha t}\right) \ln \left(\frac{4 \alpha t}{C R^{2}}\right)+\frac{R^{2}}{2 \alpha t}\right\} \text {. }
$$

Eq. 2.1.2.1 can be rewritten as follows:

$$
T(r, t)=T_{0}+\frac{R F_{0}}{2 \lambda}\left\{\left(1+\frac{R^{2}}{2 \alpha t}\right) \ln \left(\frac{4 \alpha t}{C R^{2}}\right)+\left(1+\frac{R^{2}}{4 \alpha t}\right) \ln \left(\frac{R^{2}}{r^{2}}\right)+\frac{R^{2}}{4 \alpha t}\left(1+\frac{r^{2}}{R^{2}}\right)\right\} .
$$


By noting that for large $t$,

$$
\left(1+\frac{R^{2}}{2 \alpha t}\right) \ln \left(\frac{4 \alpha t}{C R^{2}}\right)+\frac{R^{2}}{2 \alpha t} \approx \ln \left(\frac{2.34 \alpha t}{R^{2}}\right),
$$

Eq. 2.1.2.3 can be modeled by

$$
T(r, t)=T_{0}+\frac{R F_{0}}{2 \lambda}\left[\ln \left(\frac{2.34 \alpha t}{R^{2}}\right)+2 \ln \left(\frac{R}{r}\right)\right] .
$$

The "exact" equation for the temperature given by Eq. 2.1.2.1 is compared to the model given by Eq. 2.1.2.5 in Fig. 2.1.1 for the conditions given in Table 2.1.1. The model reasonably matches the exact equation except at large distances. The heat flux used was chosen so that the same net energy was transported into the formation as in the linear case. This figure also shows that the temperature profile in cylindrical geometry grows at about the same rate as that in linear geometry, although the peak temperature is higher and the temperature falls more rapidly with distance from the heat source.

The radius where a given temperature is found at a given time may be found from Eq. 2.1.2.5:

$$
r=1.53 \sqrt{\alpha t} \exp \left(-\frac{\lambda\left(T(r, t)-T_{0}\right)}{R F_{0}}\right)
$$

The position of the boiling zone is

$$
r_{b}=1.53 \sqrt{\alpha t} \exp \left(-\frac{\lambda\left(T_{b}-T_{0}\right)}{R F_{0}}\right)
$$

and the velocity of the boiling zone away from the heat source is

$$
u_{b}=\frac{d r_{b}}{d t}=0.765 \sqrt{\frac{\alpha}{t}} \exp \left(-\frac{\lambda\left(T_{b}-T_{0}\right)}{\mathrm{AF}_{0}}\right)
$$

This velocity is plotted as a function of time in Fig. 2.1.2 using the data in Table 2.1.1. From this figure, it is seen that the velocity of the advancing boiling front (dry zone) in cylindrical geometry is lower than that for linear geometry, although it is initially of the same magnitude. 


\subsubsection{Spherical Geometry}

For one-dimensional radial conduction into a medium bounded internally by a sphere with a constant heat flux at its surface, the radial temperature profile at large times can be approximated by (Carslaw and Jaeger, 1959, 248):

$$
T(r, t)=T_{0}+\frac{R^{2} F_{0}}{\lambda r}\left\{\operatorname{erfc}\left(\frac{r-R}{2 \sqrt{\alpha t}}\right)-\exp \left(\frac{r-R}{R}+\frac{\alpha t}{R^{2}}\right) \operatorname{erfc}\left(\frac{r-R}{2 \sqrt{\alpha t}}+\frac{\sqrt{\alpha t}}{R}\right)\right\},
$$

where $T_{0}$ is the initial formation temperature, $F_{0}$ is the heat flux into the formation, $\lambda$ is the formation thermal conductivity, $\alpha$ is the formation diffusivity, $t$ is the time since the start of heating, $R$ is the internal radius of the source, and $r$ is the distance into the formation from the heat source.

The temperature of the formation at the heat source is given by

$$
T(R, t)=T_{0}+\frac{R F_{0}}{\lambda}\left\{1-\exp \left(\frac{\alpha t}{R^{2}}\right) \operatorname{erfc}\left(\frac{\sqrt{\alpha t}}{R}\right)\right\} .
$$

Eq. 2.1.3.1 can be approximated by

$$
T(r, t)=T_{0}+\frac{R^{2} F_{0}}{\lambda r}\left\{1+\operatorname{erf}\left(\frac{R}{2 \sqrt{\alpha t}}\right)-25^{*} \exp \left(-\frac{\sqrt{\alpha t}}{R}\right)-\frac{\exp \left\{-\frac{R^{2}}{4 \alpha t}\right\}}{\sqrt{\pi \alpha t}} r\right\} .
$$

The "exact" equation for the temperature given by Eq. 2.1.3.1 is compared to the model given by Eq. 2.1.3.3 in Fig. 2.1.1 for the conditions given in Table 2.1.1. The model reasonably matches the exact equation. The heat flux used was chosen to that the same net energy was transported into the formation as in the linear geometry case. A rectangular grid was used for the sphere positions. This figure shows that the temperature profile in spherical geometry grows at a similar rate to that of linear and cylindrical geometry, although the peak temperature is much higher and the temperature falls much more rapidly with distance from the heat source.

The radius where a given temperature is found at a given time may be found from Eq. 2.1.3.3:

$$
r=\frac{1+\operatorname{erf}\left(\frac{R}{2 \sqrt{\alpha t}}\right)-25 \exp \left(-\frac{\sqrt{\alpha t}}{R}\right)}{\frac{\lambda\left(T(r, t)-T_{0}\right)}{R^{2} F_{0}}+\frac{\exp \left\{-\frac{R^{2}}{4 \alpha t}\right\}}{\sqrt{\pi \alpha t}}} .
$$


J. C. Reis

August 1995

The position of the boiling zone is

$$
r_{b}=\frac{1+\operatorname{erf}\left(\frac{R}{2 \sqrt{\alpha t}}\right)-25 \exp \left(-\frac{\sqrt{\alpha t}}{R}\right)}{\frac{\lambda\left(T_{b}-T_{0}\right)}{R^{2} F_{0}}+\frac{\exp \left\{-\frac{R^{2}}{4 \alpha t}\right\}}{\sqrt{\pi \alpha t}}} .
$$

The velocity of the boiling front away from the heat source is

$$
\begin{aligned}
u_{b}= & \frac{d r_{b}}{d t}=\frac{\frac{1}{2 \sqrt{t}}\left[\sqrt{\pi \alpha}-\frac{R}{\sqrt{t}} \exp \left\{-\frac{R^{2}}{4 \alpha t}\right\}+\sqrt{\pi \alpha} \operatorname{erf}\left(\frac{R}{2 \sqrt{\alpha t}}\right)\right]}{\left[\frac{\lambda \sqrt{\pi \alpha t}\left(T_{b}-T_{0}\right)}{R^{2} F_{0}}+\exp \left\{-\frac{R^{2}}{4 \alpha t}\right\}\right]} \\
+ & \frac{\frac{25}{2} \sqrt{\pi}\left[\frac{\alpha}{R}-\sqrt{\frac{\alpha}{t}}\right] \exp \left(-\frac{\sqrt{\alpha t}}{R}\right)}{\left[\frac{\lambda \sqrt{\pi \alpha t}\left(T_{b}-T_{0}\right)}{R^{2} F_{0}}+\exp \left\{-\frac{R^{2}}{4 \alpha t}\right\}\right]} \\
& -\frac{\left[1+\operatorname{erf}\left(\frac{R}{2 \sqrt{\alpha t}}\right)-25 \exp \left(-\frac{\sqrt{\alpha t}}{R}\right)\right]\left[\frac{\lambda\left(T_{b}-T_{0}\right)}{R^{2} F_{0}} \sqrt{\frac{\pi \alpha}{t}}+\frac{R^{2}}{2 \alpha t^{2}} \exp \left\{-\frac{R^{2}}{4 \alpha t}\right\}\right]}{\frac{2}{\sqrt{\pi \alpha t}}\left[\frac{\lambda \sqrt{\pi \alpha t}\left(T_{b}-T_{0}\right)}{R^{2} F_{0}}+\exp \left\{-\frac{R^{2}}{4 \alpha t}\right\}\right]^{2}} .
\end{aligned}
$$

This velocity is plotted as a function of time in Fig. 2.1.2 using the data in Table 2.1.1. From this figure, it is seen that the initial velocity of the advancing boiling front (dry zone) in spherical geometry is of the same magnitude as that of linear or cylindrical geometry and then decreases more rapidly.

\subsection{Fluid Transport}

The formation at the proposed Yucca Mountain repository is porous and permeable. Free fluids will migrate through the formation from gravity and capillary forces, as well as from the pressurization of the repository from the waste heat. In this section, analytical expressions are developed for these water transport mechanisms. 


\subsubsection{Gravity Drainage}

One of the ways that water migrates through the formation is by gravity drainage induced by differences in the density of the air and water in the formation. As water infiltrates downward through a partially-saturated formation, the air migrates upward through the water bank by counter-current flow. The geometry of a water bank draining through a formation is shown in Fig. 2.2.1. The water and flow rates can be obtained from Darcy's law:

$$
\mathrm{q}_{\mathrm{w}}=\frac{\operatorname{Akk}_{\mathrm{w}}}{\mu_{\mathrm{w}}} \frac{\left(\Phi_{\mathrm{w}, \mathrm{t}}-\Phi_{\mathrm{w}, \mathrm{b}}\right)}{\mathrm{h}}
$$

and

$$
q_{g}=\frac{A k k_{g g}}{\mu_{g}} \frac{\left(\Phi_{g, t}-\Phi_{g, b}\right)}{h},
$$

respectively, where $\Phi$ is the flow potential, the subscripts " $w$ " and " $g$ " refer to the water and gas (air) phases, respectively, and the subscripts " $t$ " and " $b$ " refer to the top and bottom of the water bank, respectively. The thickness of the water bank is " $h$ ".

The flow potential is defined as

$$
\Phi=\dot{P}+\rho g z
$$

and is used for flows in a gravitational field. The variable " $\mathrm{z}$ " is the height above an arbitrary reference plane. The flow potential accounts for the hydrostatic variation in pressure with depth that does not result in vertical flow. For this case, the flow potentials can be written as

$$
\begin{gathered}
\Phi_{w, t}=P_{w, t}+\rho_{w} g(z+h), \\
\Phi_{w, b}=P_{w, b}+\rho_{w} g z, \\
\Phi_{g, t}=P_{g, t}+\rho_{g} g(z+h),
\end{gathered}
$$

and

$$
\Phi_{g, b}=P_{g, b}+\rho_{g} g z .
$$

Inserting Eqs. 2.2.1.4a through 2.2.1.4d into Eqs. 2.2.1.1 and 2.2.1.2 yields

$$
q_{w}=\frac{A k k_{r w}}{\mu_{w}} \frac{\left[\left(P_{w, t}-P_{w, b}\right)+p_{w} g h\right]}{h}
$$


and

$$
q_{g}=\frac{A k k_{\mathrm{rg}}}{\mu_{g}} \frac{\left[\left(P_{g, t}-P_{g, b}\right)+\rho_{g} g h\right]}{h} .
$$

The gas pressures in Eq. 2.2.1.6 can be written in terms of the local water pressures and the corresponding capillary pressures:

$$
P_{g, t}=P_{w, t}+P_{c, t}
$$

and

$$
P_{g, b}=P_{w, b}+P_{c, b} .
$$

Substituting Eqs. 2.2.1.7a and 2.2.1.7b into Eq. 2.2.1.6 yields

$$
q_{g}=\frac{A k k_{r g}}{\mu_{g}} \frac{\left[\left(P_{w, t}-P_{w, b}\right)+\left(P_{c, t}-P_{c, b}\right)+\rho_{g} g h\right]}{h} .
$$

Now it is assumed that the water and gas flow rates are equal and opposite, e.g., counter-current flow of both gas and water occurs at the location of the water bank:

$$
q_{w}=-q_{g}
$$

Substituting Eqs. 2.2.1.5 and 2.2.1.8 into Eq. 2.2.1.9 and solving for the pressure difference in the water yields

$$
\left(P_{w, t}-P_{w, D}\right)=-\left[\frac{\frac{k_{r w}}{\mu_{w}} \rho_{w} g h+\frac{k_{r g}}{\mu_{g}}\left\langle\left(P_{c, t}-P_{c, b}\right)+\rho_{g} g h\right)}{\frac{k_{r w}}{\mu_{w}}+\frac{k_{r g}}{\mu_{g}}}\right] .
$$

Substituting Eq. 2.2.1.10 into Eq. 2.2.1.5 yields the following expression for the water flow rate:

$$
q_{w}=\frac{\operatorname{Ak}\left[\left(\rho_{w}-\rho_{g}\right) g h-\left(P_{c, 1}-P_{c, b}\right)\right]}{\left(\frac{\mu_{w}}{k_{r w}}+\frac{\mu_{g}}{k_{r g}}\right) h} .
$$


Eq. 2.2.1.16 is independent of time, so the distance traveled by the bank can be expressed as

$$
L=u_{w} t=\frac{k k_{w} \rho_{w} g t}{\phi \Delta S_{w} \mu_{w}} .
$$

Inverting Eq. 2.2.1.17 yields the following expression for the time it takes the bank to travel a distance "L":

$$
t^{*}=\frac{\phi \Delta S_{w} \mu_{w} L}{k k_{r w} \rho_{w} g} .
$$

Note that the velocity of the water bank and its travel time is independent of the thickness of the water bank.

To test the validity of Eq. 2.2.1.16 using for the velocity of water by gravity drainage through a geologic formation, a numerical simulation of gravity drainage of a water bank was conducted using the NUFT code with the same model as before. The initial water saturation in the numerical model was set at 0.4, except for the top 10 grids, which were set at 0.9. The resulting water saturation profiles are shown in Fig. 2.2.2 at different times. Because the NUFT results were based on a full set of relative permeability and capillary pressure data, the water saturation profile varied with time and position. Nevertheless, the velocity of the water bank obtained from Fig. 2.2.2 was within a factor of two of that calculated with Eq. 2.2.1.16 when using the relative permeability at the water saturation at the leading inflection point of the water saturation profile. This agreement is believed to be sufficiently accurate to justify the use of Eq. 2.2.1.16 to estimate the correct order of magnitude for the characteristic time for gravity drainage.

\subsubsection{Counter-Current Capillary Imbibition}

When water condenses in the fractures, it will be in capillary non-equilibrium with the water in the matrix. This will initiate spontaneous capillary imbibition of the water into the matrix.

Cil and Reis (1995) developed a model for the spontaneous counter-current capillary imbibition rate of water into a gas-saturated medium. This geometry is shown in Fig. 2.2.3. For water imbibing into a semi-infinite, planar matrix, the cumulative volume of water imbibed can be found by

$$
Q_{w}=2 A_{i m} \sqrt{\beta t},
$$

and the imbibition rate is given by 


$$
q_{w}=A_{i m} \sqrt{\frac{\beta}{t}},
$$

where

$$
\beta=\frac{\phi \Delta S k P_{c}}{2\left(\frac{\mu_{w}}{k_{r w}}+\frac{\mu_{g}}{k_{r g}}\right)} .
$$

Cil and Reis assumed that the water saturation front in the matrix advanced with a step profile, e.g., piston-like displacement. The position of the advancing water front in the matrix can be found with the following equation:

$$
x=\left[\frac{2 k P_{c} t}{\phi \Delta S\left(\frac{\mu_{w}}{k_{m}}+\frac{\mu_{g}}{k_{r g}}\right)}\right]^{1 / 2} .
$$

The time the imbibition front reaches the opposite side of the matrix block can be found by solving Eq. 2.2.2.4 for the time with $\mathrm{x}=\mathrm{L}$ :

$$
t^{*}=\frac{L^{2} \phi \Delta S\left(\frac{\mu_{w}}{K_{r w}}+\frac{\mu_{g}}{k_{r g}}\right)}{2 k P_{c}} .
$$

\subsubsection{Co-Current Capillary Imbibition}

When water spontaneously imbibes into the matrix, the air initially present will be expelled either by counter-current flow, as described above, or by co-current flow, where the air is displaced away from the imbibition front.

For the case of co-current capillary imbibition of water, imbibition is assumed to occur into one face of a matrix block and gas is expelled out of the opposite face. The pressures in the fractures at the two ends of the matrix block are assumed to be equal and the sides are impermeable. This geometry is shown in Figure 2.2.4.

The water and gas (air) flow rates through the matrix can be found from Darcy's law:

$$
\mathrm{q}_{w}=\frac{\text { Akk }_{\mathrm{rw}}}{\mu_{w}} \frac{\mathrm{dP}_{\mathrm{w}}}{\mathrm{dx}}=\frac{A k k_{\mathrm{w}}}{\mu_{w}} \frac{\left(P_{f}-P_{w, x}\right)}{x}
$$


and

$$
q_{g}=\frac{A k k_{r g}}{\mu_{g}} \frac{d P_{g}}{d x}=\frac{A k k_{r g}}{\mu_{g}} \frac{\left(P_{1}-P_{g, x}\right)}{(L-x)},
$$

respectively. In these equations, $\mathrm{P}_{\mathrm{f}}$ is the pressure in the fractures at either end of the matrix, $P_{w, x}$ is the pressure in the water at the imbibition front within the matrix block, and $\mathrm{P}_{\mathrm{g}, \mathrm{x}}$ is the gas pressure at the imbibition front. For co-current imbibition, the gas and water flow rates are related through the following expression:

$$
q_{g}=q_{w}
$$

Combining Eqs. 2.2.3.1 through 2.2.3.3 yields

$$
\frac{k_{\mathrm{rg}}}{\mu_{\mathrm{g}}} \frac{\left(\mathrm{P}_{\mathrm{f}}-\mathrm{P}_{\mathrm{g}, \mathrm{x}}\right)}{(L-\mathrm{x})}=\frac{k_{\mathrm{rw}}}{\mu_{\mathrm{w}}} \frac{\left(\mathrm{P}_{1}-\mathrm{P}_{\mathrm{w}, \mathrm{x}}\right)}{\mathrm{x}} .
$$

The gas and water pressures are related through the capillary pressure in the porous medium at the water-gas interface. This relationship is given by

$$
P_{w, x}=P_{g, x}-P_{c}
$$

Substituting Eq. 2.2.3.5 into Eq. 2.2.3.4 to eliminate the water pressure in the matrix and solving for the gas pressure yields

$$
P_{g, x}=\frac{\frac{k_{r w}}{\mu_{w}} \frac{1}{x}\left(P_{f}+P_{c}\right)+\frac{k_{r g}}{\mu_{g}}\left(\frac{1}{L-x}\right) P_{f}}{\frac{k_{m w}}{\mu_{w}} \frac{1}{x}+\frac{k_{r g}}{\mu_{g}}\left(\frac{1}{L-x}\right)} .
$$

Substituting Eq. 2.2.3.6 into Eq. 2.2.3.2 and simplifying yields the following expression for the gas expulsion rate:

$$
q_{g}=\frac{A k P_{c}}{\frac{\mu_{g}}{k_{r g}}(L-x)+\frac{\mu_{w}}{k_{r w}} x} .
$$

The gas expulsion rate from the matrix block can be found from a material balance of the amount of gas in the matrix block:

$$
Q_{g}=\int_{0}^{x} A \phi \Delta S d x
$$


or

$$
Q_{g}=A \phi \Delta S x
$$

where a step profile across the imbibition front is assumed. A step profile was observed for counter-current imbibition by Cil and Reis (1995). The gas expulsion rate is obtained by differentiating Eq. 2.2.3.9 with respect to time:

$$
q_{g}=A \phi \Delta S \frac{d x}{d t} \text {. }
$$

Equating Eqs. 2.2.3.7 and 2.2.3.10 and solving the resulting differential equation yields the following equation for the position of the imbibition front in the matrix block:

$$
x=\frac{-L+\sqrt{L^{2}+\frac{2 k k_{r g} P_{c} t}{\mu_{g} \phi \Delta S}\left(\frac{k_{r g} \mu_{w}}{k_{r w} \mu_{g}}-1\right)}}{\left(\frac{k_{r g} \mu_{w}}{k_{r w} \mu_{g}}-1\right)} .
$$

Substituting Eq. 2.2.3.11 back into Eq. 2.2.3.10 gives the following equation for the gas expulsion rate:

$$
q_{g}=\frac{A k k_{r g} P_{c}}{\mu_{g}}\left[L^{2}+\frac{2 k k_{r g} P_{c} t}{\mu_{g} \phi \Delta S}\left(\frac{k_{r g} \mu_{w}}{k_{r w} \mu_{g}}-1\right)\right]^{-\frac{1}{2}} .
$$

The cumulative volume of gas expelled can be found by substituting Eq. 2.2.3.11 into Eq. 2.2.3.9 :

$$
Q_{g}=A \phi \Delta S\left[\frac{-L+\sqrt{L^{2}+\frac{2 k_{r g} P_{c} t}{\mu_{g} \phi \Delta S}\left(\frac{k_{r g} \mu_{w}}{k_{r w} \mu_{g}}-1\right)}}{\left(\frac{k_{r g} \mu_{w}}{k_{r w} \mu_{g}}-1\right)}\right] .
$$

This equation is valid until the imbibition front reaches the opposite side of the matrix block. This time can be found by solving Eq. 2.2.3.11 for time with $x=L$ :

$$
t^{*}=\frac{L^{2} \phi \Delta S\left(\frac{\mu_{w}}{k_{r w}}+\frac{\mu_{g}}{k_{r g}}\right)}{2 k P_{c}} .
$$


By comparing Eqs. 2.2.2.5 and 2.2.3.14, it can be seen that the characteristic times for counter-current and co-current imbibition are virtually identical. It should be noted, however, that the relative permeabilities for counter-current imbibition may be somewhat lower than those for co-current imbibition because counter-current imbibition requires simultaneous flow of both phases at one point, while co-current imbibition does not.

\subsubsection{Co-Current Capillary and Gravity Imbibition}

In the previous sections, equations describing gravity drainage, counter-current spontaneous capillary imbibition, and co-current spontaneous capillary imbibition have been developed. In this section, equations for the combined mechanisms of gravity drainage and co-current imbibition will be developed.

When the density of the fluid in the fracture is different from that of the fluid in the matrix block, a hydrostatic potential difference will be established between the two fluids. This potential difference can drive fluid transfer between the matrix and fractures. When the fluid in the fracture is more dense than the fluid in the matrix block, e.g., when water is in the fractures and gas is in the matrix, then water will enter into the matrix near the bottom, forcing the gas into the fracture near the top. The geometry for this case is shown in Fig. 2.2.5.

The water flow rate into the matrix can be found from Darcy's law as

$$
q_{w}=\frac{A k k_{n w}}{\mu_{w}} \frac{d \Phi_{w}}{d z}=\frac{A k k_{w w}}{\mu_{w}} \frac{\left(\Phi_{w, b}-\Phi_{w, r}\right)}{h^{\prime}}
$$

Similarly, the flow rate of the gas out of the matrix is

$$
q_{g}=\frac{A k k_{r g}}{\mu_{g}} \frac{d \Phi_{g}}{d z}=\frac{A k k_{r g}}{\mu_{g}} \frac{\left(\Phi_{g, h^{\prime}}-\Phi_{g, h}\right)}{h-h^{\prime}},
$$

For Eqs. 2.2.4.1 and 2.2.4.2, $\Phi$ is the flow potential and is given by

$$
\Phi=P+\rho g z
$$

where $z$ increases upward. The flow potential is used in gravity drainage calculations instead of pressure to account for hydrostatic forces that do not contribute to fluid flow within an individual phase.

For this problem, the reference plane $(z=0)$ is taken as the base of the matrix block. The flow potentials are then 
J. C. Reis

$$
\begin{gathered}
\Phi_{w, b}=P_{w, b}, \\
\Phi_{w, h^{\prime}}=P_{w, h^{\prime}}+\rho_{w} g h^{\prime}, \\
\Phi_{g, h^{\prime}}=P_{g, h^{\prime}}+\rho_{g} g h^{\prime},
\end{gathered}
$$

and

$$
\Phi_{g, h}=P_{g, h}+\rho_{g} g h
$$

where the first subscript refers to the fluid and the second subscript refers to the position.

By noting that the pressure of the water in the fracture surrounding the matrix block is hydrostatic, e.g.,

$$
P_{w, b}=P_{w, h}+\rho_{w} g h
$$

the pressure of the water in the matrix block can be related to the pressure of the gas through the capillary pressure, e.g.,

$$
P_{w, W}=P_{g, \hbar r}-P_{c}
$$

the pressures of the water and gas in the fracture at the top of the matrix block are equal, e.g.,

$$
P_{g, h}=P_{w, h},
$$

and that the flow rates of water and matrix fluid are equal, e.g.,

$$
q_{w}=q_{g}
$$

Eqs. 2.2.4.1 through 2.2.4.11 can be combined to yield

$$
\left(P_{g, h^{\prime}}-P_{g, h}\right)=\frac{\frac{k_{w w}}{\mu_{w}}\left(h-h^{\prime}\right)\left[\rho_{w} g\left(h-h^{\prime}\right)+P_{c}\right]+\frac{k_{r g}}{\mu_{g}} h^{\prime}\left[\rho_{g} g\left(h-h^{\prime}\right)\right]}{\left[\frac{k_{r w}}{\mu_{w}}\left(h-h^{\prime}\right)+\frac{k_{r g}}{\mu_{g}} h^{\prime}\right]} .
$$


Combining Eq. 2.2.4.12 with Eqs. 2.2.4.2, 2.2.4.6 and 2.2.4.7 yields the following equation for the expulsion rate for the gas in the matrix:

$$
q_{g}=A k\left[\frac{\left(\rho_{w}-\rho_{g}\right) g\left(h-h^{\prime}\right)+P_{c}}{\frac{\mu_{w}}{k_{r w}} h^{\prime}+\frac{\mu_{g}}{k_{r g}}\left(h-h^{\prime}\right)}\right] .
$$

A second expression for the gas expulsion rate can be obtained from a material balance on the fluid. The expulsion rate.can be written as

$$
q_{g}=A \phi \Delta S \frac{d h^{\prime}}{d t} .
$$

Equating Eqs. 2.2.4.13 and 2.2.4.14 yields the following nonlinear differential equation:

$$
A \frac{d h^{\prime}}{d t}+B h^{\prime} \frac{d h^{\prime}}{d t}+C h^{\prime}=D \text {, }
$$

where

$$
\begin{gathered}
A=\frac{\phi \Delta S \mu_{g} h}{k k_{r g}}, \\
B=\frac{\phi \Delta S}{k}\left(\frac{\mu_{w}}{k_{r w}}-\frac{\mu_{g}}{k_{r g}}\right), \\
C=\left(\rho_{w}-\rho_{g}\right) g
\end{gathered}
$$

and

$$
D=\left(\rho_{w}-\rho_{g}\right) g h+P_{c}
$$

This differential equation has the following solution:

$$
t=-\frac{A C+B D}{C^{2}} \ln \left(1-\frac{C}{D} h^{\prime}\right)-\frac{B}{C} h^{\prime},
$$

or in terms of primary variables, 


$$
t_{D}=\left\{\frac{h^{\prime}}{h}(M-1)-\left[1-\frac{P_{c}}{\left(\rho_{w}-\rho_{g}\right) g h}(M-1)\right] \ln \left[1-\frac{\left(\rho_{w}-\rho_{g}\right) g}{\left(\rho_{w}-\rho_{g}\right) g h+P_{c}} h^{\prime}\right]\right\},
$$

where a dimensionless time has been defined as

$$
t_{D}=\frac{k k_{w w}\left(\rho_{w}-\rho_{g}\right) g t}{\phi \Delta S \mu_{w} h}
$$

and a mobility ratio has been defined as

$$
M=\frac{\mu_{g} k_{r w}}{k_{r g} \mu_{w}} .
$$

For the case of no capillary pressure, e.g., pure gravity drainage, Eq. 2.2.4.21 simplifies to

$$
t_{D}=\frac{h^{\prime}}{h}(M-1)-\ln \left(1-\frac{h^{\prime}}{h}\right) .
$$

If a characteristic time for gravity drainage is defined as the time required for drainage to reach a completion of about $63 \%, e . g ., h / h^{\prime}=1-1 / e$, where $e$ is the base of the natural logarithm scale, then the characteristic time for gravity drainage can be obtained by evaluating Eq. 2.2.4.24 at the selected point and combining it with Eq. 2.2.4.22:

$$
t^{*}=\frac{\phi \Delta S \mu_{w} h}{k_{r w}\left(\rho_{w}-\rho_{g}\right) g}\left[\left(1-\frac{1}{e}\right)\left(\frac{\mu_{g} k_{r w}}{k_{r g} \mu_{w}}-1\right)+1\right] .
$$

\subsubsection{Effect of Crossflow on Water Advance in Fractures}

When some of the water flowing through fractures is imbibed into the surrounding matrix, the distance that water penetrates through the fracture network is reduced. If the rate of water loss from the fractures to the matrix is known, the penetration distance of water into the fracture network can be determined from a material balance.

In this section, an equation for the position of the water front in a high-permeability layer when capillary crossflow to adjacent layers occurs is developed. Water is injected into the high-permeability layer and piston-like displacement in that layer is assumed. Water imbibes from this layer into the adjacent low-permeability layers. The adjacent layers are assumed to be infinitely thick, e.g., the imbibition front does not reach a far boundary during the time of interest. Gravitational forces are neglected. The three layer geometry used is shown in Figure 2.2.6.

A material balance on the injected water yields the following equation: 


$$
q_{1}=q_{a c c}+q_{\text {loss }}
$$

where $q_{i}$ is the water injection rate into the high-permeability layer (fracture), $q_{a c c}$ is the rate at which water accumulates in that layer, and qloss is the rate of water loss to the adjacent layers by capillary crossflow.

The rate at which water accumulates in the layer can be expressed by

$$
q_{a c c}=h \phi \Delta S_{\text {lay }} \frac{d A_{m}}{d t},
$$

where $h$ is the layer thickness, $\phi$ is its porosity, $\Delta S_{\text {lay }}$ is the change in water saturation in the layer at the leading edge of the water-swept area, and $A_{i m}$ is the area that has been penetrated by water, e.g., the wetted area of the high-permeability layer in contact with one of the adjacent layers. This is the area through which imbibition to one of the adjacent layers occurs.

The rate of water loss by capillary crossflow to the adjacent layers is given by

$$
q_{\text {loss }}=2 \int_{0}^{A_{m}} u(t-\gamma) d A_{l m},
$$

where $u$ is the local capillary crossflow velocity in each layer and $\lambda$ is the time the water front in the high-permeability layer passes the incremental area $\mathrm{dA}$ im. Thus, $\mathrm{t}-\gamma$ is the time that water has been imbibing into the incremental area.

The capillary crossflow rate given by Eq. 2.2.2.2 can be used to determine the local capillary crossflow velocity. The local velocity is given by

$$
u(t-\gamma)=\frac{\delta q_{w}}{\delta A_{w m}}=\left[\frac{\beta}{(t-\gamma)}\right]^{1 / 2},
$$

where $\beta$ is given by Eq. 2.2.2.3. Combining Eqs. 2.2.5.1 through 2.2.5.4 yields the following integro-differential equation for the water swept area of the layer:

$$
q_{1}=h \phi \Delta S_{\text {lay }} \frac{d A_{l m}}{d t}+2 \int_{0}^{A_{m}}\left[\frac{\beta}{(t-\gamma)}\right]^{1 / 2} d A_{l m} .
$$

The integral in this equation can be converted from one over area to one over time, yielding 
J. C. Reis

$$
q_{1}=h \phi \Delta S_{\text {lay }} \frac{d A_{\text {lm }}}{d t}+2 \int_{0}^{1}\left[\frac{\beta}{(t-\gamma)}\right]^{1 / 2} \frac{d A_{l m}}{d \gamma} d \gamma
$$

The LaPlace transform of Eq. 2.2.5.6 is

$$
\frac{q_{i}}{s}=h \phi \Delta S_{\text {lay }} s A_{i m}(s)+2 \sqrt{\pi \beta} \sqrt{s} A_{i m}(s) .
$$

Solving this equation for the area in LaPlace space and taking the inverse LaPlace transform of the result gives the following equation for the area of the high-permeability layer swept by water:

$$
A_{\text {tm }}(t)=\frac{q h \phi \Delta S_{\text {lay }}}{4 \pi \beta}\left[\exp \left\{\frac{4 \pi \beta t}{\left(h \phi \Delta S_{\text {lay }}\right)^{2}}\right\} \operatorname{erfc}\left\{\frac{2 \sqrt{\pi \beta t}}{h \phi \Delta S_{\text {lay }}}\right\}+\frac{4 \sqrt{\beta t}}{h \phi \Delta S_{\text {lay }} \sqrt{\pi}}-1\right] .
$$

For the case of the high-permeability layer being a fracture, e.g., in the limit as the layer thickness $(h)$ goes to zero, Eq. 2.2.5.8 simplifies to

$$
A_{m}(t)=\frac{q_{i}}{\pi} \sqrt{\frac{t}{\beta}} .
$$

The position of the front in the fracture can be obtained from the surface area through the following equation:

$$
x(t)=\frac{q}{\pi W} \sqrt{\frac{t}{\beta}},
$$

where $W$ is the width of the fracture (perpendicular to the aperture and flow direction).

The velocity of the water front through the fracture in the presence of capillary imbibition is found by differentiating Eq. 2.2.5.10 and substituting in Eq. 2.2.2.3:

$$
u_{t}(t)=\frac{q_{i}}{\pi W} \sqrt{\frac{\left(\frac{\mu_{w}}{k_{r w}}+\frac{\mu_{g}}{k_{r g}}\right)}{2 \phi \Delta S k P_{c} t}} .
$$

\subsubsection{Formation Pressurization From Heating}

When the formation is heated by conduction above the boiling temperature of water, the liquid water in the formation will undergo a phase transformation to the vapor phase. This phase transformation is accompanied by a large increase in specific 
volume. This increase in specific volume results in an increase in the pressure in the formation, which drives fluids away from the repository and relieves the pressure.

If the formation permeability is low, the pressure in the dry zone can increase substantially. This increase in pressure will also increase the boiling temperature, minimizing further vaporization. In this case, the temperature around the repository would continue to increase without the formation of a significant dry zone.

The pressurization of the matrix from thermal conduction was studied numerically using NUFT using the same model as before. As seen in Fig. 2.2.7, this pressurization can occur at permeabilities on the order of microdarcys. Since the matrix permeability is on the order of microdarcys, whether or not the matrix will pressurize and raise the boiling point will depend primarily on the fracture spacing.

Reis (1992) developed a procedure for evaluating the pressure diffusion rate in a matrix block bounded by permeable fractures. He calculated the average pressure in a gas-saturated matrix block as a function of time after the pressure in the adjacent fractures is instantaneously dropped to a lower value. The square of the average matrix pressure was reported to be

$$
\bar{P}^{2}(t)=P_{1}^{2}+\frac{8\left(P_{1}^{2}-P_{1}^{2}\right)}{\pi^{2}} \exp \left\{-\frac{\pi^{2} k k_{r g} P_{\text {avg }} t}{\phi \mu_{g} L^{2}}\right\},
$$

where $P_{j}$ is the initial pressure in the matrix block, $P_{f}$ is the pressure in the fracture, $k_{r g}$ is the effective permeability of the matrix block, $P_{\text {avg }}$ is an average pressure of the system, $\phi$ is the porosity of the matrix block, $\mu_{\mathrm{g}}$ is the viscosity of the gas, and $L$ is the fracture spacing (width of the matrix block).

The characteristic time for the matrix to be depressurized is

$$
t^{*}=\frac{\phi \mu_{g} L^{2}}{\pi^{2} k k_{\text {rg }} P_{\text {avg }}} .
$$

For the conditions listed in Table 2.2.2, the characteristic time for matrix depressurization is plotted in Fig. 2.2.8 as a function of fracture spacing. For expected fracture spacings on the order of meters or less, the characteristic time for the matrix to depressurize is on the order of days. Because of the relatively slow velocity of the boiling front, significant pressurization is not expected to occur unless the fracture spacing is very high. If the local fracture spacing is very high, the pressure can increase to where it exceeds the tensile strength of the rock. This would create fractures in the rock to increase the fracture spacing and allow the matrix to depressurize. 


\subsection{Creation of a Water Bank}

As the repository heats, the temperature around individual waste packages can exceed the boiling temperature. When this occurs, the water trapped in the pores by capillary pressure will be vaporized and transported out of the growing dry zone, where it subsequently condenses. The condensed water above the repository either remain in the formation above the repository or drain away from the repository from gravitational forces. If the water remains above the repository, it constitutes a potential threat to the integrity of the repository containment after the nuclear heating has decayed and the formation cools.

If the water remains above the repository, it will either remain in the fracture network or enter the matrix. Because the fracture porosity is low, the amount of water stored in the fractures is low and the threat to the repository is low. A significant water bank can exist only if water enters the matrix above the repository.

\subsection{Height of Water Bank}

The height of the water bank above the dry zone can be determined from a material balance on the water. Initially the formation is at its initial water saturation, $\mathrm{S}_{\mathrm{wi}}$. As the boiling front approaches, the water saturation in the bank increases to its maximum saturation, $\mathrm{S}_{\mathrm{wm}}$. The maximum saturation within this water bank may be less than unity to allow for a trapped gas (air) saturation in the formation. Behind the boiling front, the water saturation is zero. Actually a steam plateau may exist behind the boiling front with a declining water saturation into the dry zone. In this analysis, the width of any steam plateau is assumed to be small, maximizing the amount of water in the bank. The idealized water saturation profile is shown schematically in Fig. 3.1.1.

The position of the leading edge of the water bank, e.g., the fluid front, is found from the position of the boiling front:

$$
x_{1}=x_{b}+\Delta x,
$$

where $x_{f}$ is the position of the leading edge of the water bank, $x_{b}$ is the position of the boiling front, and $\Delta x$ is the height of the water bank.

For linear geometry, the flow rate of water into the water bank can be found from the volumetric expansion rate of the boiling zone:

$$
q_{i n}=A \phi_{1} S_{w 1} \frac{d x_{b}}{d t}=A \phi_{1} S_{w 1} u_{b} .
$$

where $\phi_{i}$ is the porosity of the formation containing the initial water saturation.

The incremental amount of water in a water bank, e.g., the volume of water above its initial volume, is 


$$
Q_{b}=A \phi_{1} S_{w b} x_{b} \text {, }
$$

where $S_{\text {wib }}$ is the initial water saturation in the part of the formation containing the water bank. The height of the water bank above the boiling front is

$$
\Delta x=\frac{Q_{b}}{A \phi_{w b}\left(S_{w m}-S_{w b}\right)},
$$

or after substituting Eq. 3.1.3 for the volume of water,

$$
\Delta x=\frac{\phi_{1} S_{w i}}{\phi_{w b}\left(S_{w m}-S_{w b}\right)} x_{b}
$$

where $\phi_{\mathrm{wb}}$ is the porosity of the formation containing the water bank.

If the water in the bank remains in the fracture network, the porosity holding the water $\left(\phi_{w b}\right)$ is the fracture porosity. In this case, the maximum water saturation in the bank will be nearly unity and the initial saturation in the bank will be nearly zero. For a fracture porosity of 0.001 , a matrix porosity $\left(\phi_{i}\right)$ of $11 \%$, and an initial water saturation in the formation of $68 \%$, the height of the water bank in the fractures would be nearly two orders of magnitude greater than the distance from the repository to the boiling front, $x_{b}$. Since the dry zone for can be several hundred meters high (Buscheck and Nitao, 1993), the maximum value of $x_{b}$ (one-half of the total height of the dry zone) can be 100 meters. If the water remains only in the fractures, then the height of the water bank would be on the order of 10,000 meters. Since the repository itself is only a few hundred meters below the surface of the earth, the water in the fractures would be expelled from the formation and flow away from Yucca Mountain in surface topological drainage features. Only a very small fraction of the water entering the bank would remain in the formation above the repository. Thus, a water bank above the repository that exists only in the fracture network is not considered a threat to the repository integrity.

If the water above the repository in the bank enters the matrix, the porosities in Eq. 3.1 .5 are virtually equal, the maximum water saturation in the water bank will probably be around $95 \%$ to allow for a trapped air saturation in the matrix, and the initial water saturation will still be about $68 \%$. In this case, the thickness of the water bank above the dry zone will be roughly equal to $x_{b}$. This estimated height is comparable to that simulated by Buscheck and Nitao (1993) using the equivalent continuum model, e.g., capillary equilibrium between the fractures and matrix.

The height of a water bank in the fractures in the presence of non-equilibrium capillary imbibition into the matrix can be approximated by using the equation for water front velocity in the fractures in the presence of capillary cross flow: Eq. 2.2.5.10.

Substituting the water flow rate into the fractures given by Eq. 3.1.2 and 2.1.1.6 into Eq. 2.2.5.10 yields 


$$
\Delta x=\frac{A \phi_{1} S_{w i}}{2 \pi W} \sqrt{\frac{2 \alpha\left(\frac{\mu_{w}}{k_{r w}}+\frac{\mu_{g}}{k_{r g}}\right)}{\phi \Delta S_{m} k P_{c}}}\left[1+\ln \left(\sqrt{\frac{\alpha t}{\pi}} \frac{2 F_{0}}{\lambda\left(T_{b}-T_{0}\right)}\right)\right],
$$

The area through which the water is displaced is the fracture width (perpendicular to the aperture and flow direction), $W$, times the fracture spacing, "a":

$$
A=W a \text {. }
$$

Substituting Eq. 3.1.7, into Eq. 3.1.6 yields

$$
\Delta x=\frac{a S_{w 1}}{2 \pi} \sqrt{\frac{2 \phi \alpha\left(\frac{\mu_{w}}{k_{r w}}+\frac{\mu_{g}}{k_{r g}}\right)}{\Delta S_{m m} k P_{c}}}\left[1+\ln \left(\sqrt{\frac{\alpha t}{\pi}} \frac{2 F_{0}}{\lambda\left(T_{b}-T_{0}\right)}\right)\right] .
$$

Using the data in Table 3.1.1, the height of the water bank in the fractures was calculated for several fracture spacings and plotted in Fig. 3.1.2. From this figure, it can be seen that the height of the water bank in the fractures varies with fracture spacing. For a fracture spacing on the order of less than ten meters, the height of the water bank is on the order of $100 \mathrm{~m}$ or less. Because this is the same order as the height of a water bank in the matrix, a water bank in the fracture network significantly higher than that in the matrix is not expected to be present. The water would imbibe into the matrix as fast as it condenses in the fracture from the expanding dry zone. Water will not be driven to the surface through the fracture network. The imbibition rate will be discussed in more detail below.

\subsection{Vertical Gravity Drainage}

Once a water bank starts to form, it will be at a saturation above the trapped water saturation and will be mobile. This water will then drain away from the repository. If the gravity drainage rate is slow relative to other water transport rates, then a water bank could grow above the repository. However, if the gravity drainage rate is rapid relative to other transport rates, e.g., the capillary imbibition rate, then the water would drain away from the repository as quickly as it condenses instead of imbibing into the matrix. In this case, the water saturation will not build up in the matrix to form a significant bank and the integrity of the repository will not be compromised by rewetting from a water bank.

The velocity of water draining through the formation can be estimated using Eq. 2.2.1.16. For the properties given in Table 3.2.1, the velocity of water draining downward through the bulk formation would about 1000 meters/year. The saturation change used is based on an initial matrix saturation of $68 \%$ and a maximum matrix saturation in a water bank of $95 \%$. The bulk permeability of the formation for this case was 280 millidarcys. 
The gravity drainage velocity of 1000 meters/year can be compared to the velocity of the growing dry zone given in Fig. 2.1.2. From this figure, it is seen that the maximum boiling front velocity is on the order of one meter/year. The velocity of water draining downward through the formation by gravity drainage is at least three orders of magnitude greater than the velocity of the boiling front. Thus, water entering the bank from the expanding dry zone will drain away very rapidly relative to the condensation rate.

The gravity drainage velocity for water through the formation at Yucca Mountain is actually expected to be much greater that estimated using the bulk permeability of 280 millidarcys given in Table 3.2.1. When water vapor is driven from the dry zone, it condenses in the fractures. The water then drains primarily through the fracture network, not the bulk formation. Thus, a more realistic estimate of the drainage velocity is obtained if the fracture permeability is used in Eq. 2.2.1.16.

For a system of uniformly-spaced parallel fractures, the bulk formation permeability can be related to the intrinsic matrix and fracture permeabilities through the following equation:

$$
k_{b}=\frac{a k_{m}+b k_{1}}{a+b},
$$

where " $a$ " is the matrix block size, "b" is the effective fracture aperture, and $k_{m}$ and $k_{f}$ are the intrinsic permeabilities of the matrix and fracture, respectively. The intrinsic permeability is the permeability obtained from Darcy's law using only the cross sectional area of the matrix or fracture, instead of the cross sectional area of the bulk formation.

Because the matrix block size is orders of magnitude greater than the fracture aperture, Eq. 3.2.1 can be simplified to

$$
k_{b}=k_{m}+\frac{b}{a} k_{1} .
$$

Further, the matrix permeability is normally orders of magnitude lower than the bulk permeability (microdarcys vs. millidarcys), so it contributes very little to the bulk permeability. Thus,

$$
k_{b}=\frac{b}{a} k_{i} .
$$

Because the fracture porosity can be written as

$$
\phi_{1}=\frac{b}{a+b} \approx \frac{b}{a},
$$

Eq. 3.2.3 can be written as 


$$
k_{b}=\phi_{1} k_{1} .
$$

Although the fracture porosity is not known, virtually all fractured formations have fracture porosities less than $0.5 \%$ (Nelson, 1985). If the fracture porosity is assumed to be $0.1 \%$, then the intrinsic fracture permeability is one thousand times greater than the bulk permeability. Thus, for a bulk permeability of 280 millidarcys, the intrinsic fracture permeability is 280 darcys and the gravity drainage velocity through fractures is one thousand times faster than that estimated for the bulk formation. For a fracture permeability of 280 darcys, the velocity of water draining down the fractures would be one million meters/year. Thus, the estimated gravity drainage velocity through the fracture system is six orders of magnitude greater than the velocity of the boiling front.

From this analysis, it is concluded that the drainage velocity of water is many orders of magnitude faster than the velocity of the boiling zone. Thus, any water entering a water bank from the expanding dry zone will drain away far more rapidly than it will build up in the fractures. The capillary imbibition rate of water into the matrix is discussed below.

\subsection{Gravity Drainage Around Dry Zone}

In the previous section, it was assumed that water drained vertically at the velocity given by Eq. 2.2.1.16. However, because the dry zone is above the boiling temperature of water, liquid water cannot drain through it, but must drain around it. The local velocity of water draining around the edge of the dry zone is

$$
u_{L}=\frac{k k_{m} \rho_{w} g}{\phi \Delta S_{w} \mu_{w}} \cos (\theta) .
$$

where $\theta$ is the angle between the direction of fluid flow and downward vertical. For water near the top of the dry zone, the angle is near 90 degrees and the velocity is near zero. For water near the sides of the dry zone, the angle is near zero and the velocity approaches that of Eq. 2.2.1.16. The vertical component of the local velocity for gravity drainage is

$$
u_{w}=\frac{k k_{m w} \rho_{w} g}{\phi \Delta S_{w} \mu_{w}} \cos ^{2}(\theta) .
$$

Since the average angle between the direction of flow and vertical for a cylindrical or spherical dry zone will be about 45 degrees, the average vertical velocity will be about one half of the velocity of water draining vertically. This means that the time for water to drain around a cylindrical or spherical dry zone will still be of the same order as that for vertical gravity drainage.

It is then concluded that Eq. 2.2.1.16 can be used for the gravity drainage velocity around dry zones around individual waste packages or drifts For this case, the gravity 
drainage rate will remain many orders of magnitude greater than the velocity of the individual expanding dry zones.

Over time, however, the individual dry zones will merge to form a continuous dry zone across the entire repository. This continuous dry zone will have a horizontal dimension much larger than the vertical dimension. In this case, the average angle between the direction of fluid flow around the periphery of the dry zone and the downward vertical will increase, reducing the vertical component of velocity and increasing the time required for water to drain around the dry zone.

The time required for individual dry zones to merge into a continuous dry zone can be estimated from the water mobilization rate and the enthalpy required to vaporize the mobilized water. The individual dry zones will merge when the rate of horizontal heat conduction is sufficient to vaporize all of the mobilized water that is draining between the individual dry zones around waste packages.

The water drainage rate can be estimated from the volumetric growth rate of the dry zone. For a planar dry zone (linear geometry), the amount of water mobilized from the expanding dry zone can be approximated by

$$
q_{w}=L y \phi S_{w 1} u_{b},
$$

where $L$ is the spacing between drifts in the repository and $y$ is a distance along a drift.

For the boiling front velocity given by Eq. 2.1.1.6, the water mobilization rate can be written as

$$
\mathrm{q}_{w}=\frac{\operatorname{Ly} \phi S_{w i}}{2} \sqrt{\frac{\alpha}{t}}\left[1+\ln \left(\sqrt{\frac{\alpha t}{\pi}} \frac{2 F_{0}}{\lambda\left(T_{b}-T_{0}\right)}\right)\right] .
$$

The enthalpy required to vaporize this draining water is

$$
q_{v a p}=q_{w} \rho_{w} h_{\text {tg }} \text {, }
$$

or

$$
q_{\text {vap }}=\frac{{\operatorname{Ly} \phi S_{w} \rho_{w} h_{1 g}}_{2}}{\frac{\alpha}{t}}\left[1+\ln \left(\sqrt{\frac{\alpha t}{\pi}} \frac{2 F_{0}}{\lambda\left(T_{b}-T_{0}\right)}\right)\right],
$$

where $h_{\mathrm{fg}}$ is the latent heat of water.

The enthalpy required to vaporize the draining water is provided by thermal conduction from the dry zone. The horizontal heat conduction rate to water draining between individual dry zones can be approximated as 


$$
q_{h}=2\left[\lambda 2 x_{b} y \frac{\left(T_{c}-T_{b}\right)}{\left(\frac{1}{2} L\right)}\right],
$$

where $T_{c}$ is the characteristic temperature of the dry zone driving conduction, $T_{b}$ is the boiling temperature of the draining water, and $x_{b}$ is the half-height of the dry zone and is given by Eq. 2.1.1.5. The first " 2 " in the equation accounts for conduction from the two dry zones on either side of the draining water, the second " 2 " converts the halfheight of the dry zone to the total height above and below the repository, and the "1/2" indicates that conduction occurs from the center of a dry zone to its edge.

The characteristic temperature is assumed to be the average temperature of the dry zone in a vertical plane passing through a drift. This temperature can be approximated using Eq. 2.1.3 and is:

$$
T_{c}=\frac{1}{x_{b}} \int_{0}^{x_{b}}\left[T_{0}+\frac{2 F_{0}}{\lambda} \sqrt{\frac{\alpha t}{\pi}} \exp \left\{-\frac{x}{\sqrt{\alpha t}}\right\}\right] d x,
$$

or

$$
T_{c}=T_{0}+\frac{2 F_{0} \alpha t}{x_{b} \lambda \sqrt{\pi}}\left[1-\exp \left\{-\frac{x_{b}}{\sqrt{\alpha t}}\right\}\right] .
$$

Substituting Eqs. 3.3.9 and 2.1.1.5 into Eq. 3.3.7 yields the following expression for the horizontal heat conduction rate:

$$
\mathrm{q}_{\mathrm{h}}=\frac{8 \lambda y}{\mathrm{~L}} \sqrt{\alpha \mathrm{t}}\left(\mathrm{T}_{\mathrm{b}}-\mathrm{T}_{0}\right)\left\{\sqrt{\frac{\alpha t}{\pi}} \frac{2 \mathrm{~F}_{0}}{\lambda\left(\mathrm{T}_{\mathrm{b}}-\mathrm{T}_{0}\right)}-\ln \left[\sqrt{\frac{\alpha t}{\pi}} \frac{2 \mathrm{~F}_{0}}{\lambda\left(\mathrm{T}_{\mathrm{b}}-\mathrm{T}_{0}\right)}\right]-1\right\} .
$$

The enthalpy required to vaporize all of the mobilized water, given by Eq. 3.3.6, and the enthalpy transported to the mobilized water by horizontal heat conduction, given by Eq. 3.3.10, are plotted in Fig. 3.3.1 using the data in Table 3.3.1. For these data, the time required for the formation to reach the boiling temperature and start creating a dry zone is about 3 years. The horizontal heat conduction rate will exceed the enthalpy required to vaporize the mobilized water after about 15 years. The half-height of the linear geometry dry zone at that time is 13 meters. This time is of the same order as that simulated by Buscheck and Nitao (1994) using an equivalent continuum model.

In the above analysis, a drift spacing containing waste packages was used with a planar dry zone (linear geometry) to determine when the horizontal heat conduction rate would exceed the enthalpy required to vaporize the mobilized water. This seemingly conflicting geometry of a planar dry zone with individual drifts yields a liberal estimate of how long it would take for individual dry zones to merge. The linear geometry for the expanding dry zone yields the highest water mobilization rate. The 
calculated water mobilization rate assumed that all of the enthalpy provided by the heat source goes to the expanding dry zone and none went to horizontal heat conduction to vaporize the mobilized water. Because some of the decay heat will go to horizontal heat conduction, the actual water mobilization rate will be much less than that given by Eq. 3.3.4 and the enthalpy required to vaporize this water will be much less than that given by Eq. 3.3.6. The horizontal heat conduction rate given by Eq. 3.3.7 is actually less than that expected because the average temperature was calculated with the linear geometry model. The actual characteristic temperature in the dry zone will be closer to that calculated using the cylindrical geometry model. Because the characteristic temperature for cylindrical geometry will be higher than that for linear geometry, the horizontal heat conduction rate will be underpredicted by Eq. 3.3.10.

Thus, the above analysis overpredicts the water mobilization rate and underpredicts the horizontal conduction rate. This results in the time required for the individual dry zones to merge to be overpredicted. The actual time for individual dry zones to merge and create a continuous dry zone around the entire repository is expected to be less than the calculated 15 years.

It is concluded that the individual dry zones will probably merge very early in the life of the repository. Thus, the water mobilized by the growing dry zone will likely drain around the periphery of the entire repository. The time for mobilized water to drain around the repository must then be considered when determining if a water bank will be created.

If the continuous dry zone around the entire repository is modeled as having a triangular shape, as shown in Fig. 3.3.2, the average drainage angle can be approximated through

$$
\tan (\theta)=\frac{L_{r}}{x_{b}},
$$

or

$$
\cos (\theta)=\frac{x_{b}}{\sqrt{x_{b}^{2}+L_{r}^{2}}},
$$

where $L_{r}$ is the half radius of the dry zone around the repository and $x_{b}$ is the half height of the dry zone at the center of the repository.

For a repository area of $A_{r}$, the half width of the dry zone can be approximated as

$$
L_{r}=\sqrt{\frac{A_{r}}{\pi}} .
$$

Combining Eqs. 3.3.11 through 3.3.13 with Eq. 3.3.2 yields the following average vertical velocity of the water draining around the continuous dry zone: 


$$
u_{w}=\frac{k k_{m} \rho_{w} g}{\phi \Delta S_{w} \mu_{w}}\left(\frac{x_{b}^{2}}{x_{b}^{2}+L_{r}^{2}}\right)
$$

or

$$
u_{w}=\frac{k k_{m} \rho_{w} g}{\phi \Delta S_{w} \mu_{w}}\left(\frac{1}{1+\frac{A_{r}}{\pi x_{b}^{2}}}\right) .
$$

Assuming a maximum half-height of the dry zone, $x_{b}$, of 150 meters, the ratio of the drainage velocity around a continuous dry zone (Eq. 3.3.15) to the vertical velocity (Eq. 2.2.1.16) is shown in Fig. 3.3.3 as a function of repository area. For a repository size of a few thousand acres, the average vertical velocity for the water flowing around the continuous dry zone is two orders of magnitude lower than that of water draining vertically.

For gravity drainage through fractures, the downward velocity of water flowing around a continuous dry zone is still many orders of magnitude greater than the upward velocity of the boiling front. Thus, the majority of the water entering the formation above the repository from the growing dry zone can be expected to drain around and away from the repository virtually a quickly as it condenses above the dry zone.

\subsection{Matrix Imbibition Rate}

For a significant water bank to exist above the repository, water must imbibe into the matrix from the fractures at a rate faster than it drains away from the repository from gravitational forces.

The spontaneous imbibition rate has been measured for volcanic tuff similar to that at the Large Block Test at Yucca Mountain (Roberts, 1994: Lin, 1994). The apparatus used was similar to that shown in Fig. 2.2.5, except that the water head was about 1 $\mathrm{cm}$ higher than the top of the 4 inch block, as shown in Figure 3.4.1 In this test, the combined mechanisms of capillary imbibition and gravity drainage were measured.

The measured imbibition data are plotted in Figure 3.4.2. From this figure, it can be seen that the measured imbibition rate is on the order of 1000 hours per foot or about two meters per year. Comparing this imbibition velocity to the velocities of the boiling front given in Fig. 2.1.2 reveals that the velocity of water imbibing into the matrix from the combined capillary and gravity forces is the same order as the maximum velocity of the boiling front. Because of this, water imbibing into the matrix immediately above the boiling front will be reboiled virtually as fast as it imbibes. Thus, the water will not have sufficient time to imbibe into the matrix to create a water bank in the matrix, unless a water bank exists in the fractures at a height well above the boiling front. 
The experimental data were compared with the various imbibition models described in this report and are shown in Fig. 3.4.2. These models include the combined co-current capillary and gravity imbibition, gravity imbibition (without capillarity), co-current imbibition (without gravity), and counter-current imbibition (without gravity) given by Eqs. 2.2.4.21, 2.2.4.24, 2.2.3.11, and 2.2.2.4, respectively. The petrophysical data used in the figure are listed in Table 3.4.1. The capillary pressure was not measured but was varied until a match to the imbibition rate was obtained using a matrix permeability of one microdarcy.

From Fig. 3.4.2, it can be seen that the three different equations for capillary imbibition were virtually identical for these conditions and matched the measured data. It can also be seen that the imbibition rate for gravitational forces is very small relative to capillary forces and can be neglected. This figure validates the use of these capillary imbibition models without gravity for estimating the imbibition rate from fractures into the matrix.

The velocity of the water front in a fracture in the presence of imbibition into the surrounding matrix can be found with Eq. 2.2.5.11. By using the water flow rate into the fractures given by Eq. 3.1.2 and the boiling front velocity given by Eq. 2.1.1.6, the following expression is obtained for the velocity of the water front in the fractures:

$$
u_{f}=\frac{A \phi_{1} S_{w i}}{2 \pi W t} \sqrt{\frac{\alpha\left(\frac{\mu_{w}}{k_{r w}}+\frac{\mu_{g}}{k_{r g}}\right)}{2 \phi \Delta S_{m} k P_{c}}}\left[1+\ln \left(\sqrt{\frac{\alpha t}{\pi}} \frac{2 F_{0}}{\lambda\left(T_{b}-T_{0}\right)}\right)\right],
$$

The area through which the water flows is the fracture width (perpendicular to the aperture and flow direction), W, times the fracture spacing, "a":

$$
A=W a \text {. }
$$

Substituting Eq. 3.4.2, into Eq. 3.4.1 yields

$$
u_{f}=\frac{a \phi_{1} S_{w 1}}{2 \pi t} \sqrt{\frac{\alpha\left(\frac{\mu_{w}}{k_{m}}+\frac{\mu_{g}}{k_{m g}}\right)}{2 \phi \Delta S_{m} k P_{c}}}\left[1+\ln \left(\sqrt{\frac{\alpha t}{\pi}} \frac{2 F_{0}}{\lambda\left(T_{b}-T_{0}\right)}\right)\right],
$$

The velocity of the water in the fractures is plotted in Fig. 3.4.3 for several fracture spacings using the data in Table 3.1.1. For most of the life of the repository, the velocity of water in the fractures in the presence of imbibition will be on the order of 1 meter/year or less for fracture spacings less than $10 \mathrm{~m}$.

\subsection{Discussion of Water Bank}

In this section, the characteristic fluid velocities for different transport mechanisms have been compared. It was seen that the gravity drainage velocity in the absence of 
capillary imbibition was many orders of magnitude higher than the velocity of the boiling front and the imbibition velocity of water from fractures into the matrix. It was also seen that the velocity of the water front in fractures in the presence of capillary imbibition when driven by an expanding dry zone is of the same order as the capillary imbibition velocity.

Based on the analysis of this section, the following model of water migration is presented.

Water condensing in fractures below the repository will drain downward from gravity and imbibe into the matrix as it passes. The downward velocity for this water can be approximated by Eq. 3.4.3 and the height (thickness) of the water bank in the fractures below the repository can be approximated by Eq. 3.1.8.

For water condensing in the fractures above the repository, however, a more complex condition occurs. First, water will condense everywhere around the periphery of the dry zone. Some of this condensate will locally imbibe into the matrix creating a thin halo of water in the matrix around the dry zone where the water condenses. Water not imbibing into this halo will drain by gravity along the periphery of the dry zone until is flows past the edge of the dry zone and away from the repository. Because the water saturation in the matrix within the halo will already be high from the imbibition of water that condensed locally, further imbibition of water draining along the periphery will be minimal. Because the imbibition velocity of water is of the same order as that of the upward-moving boiling front, little water can imbibe upward to thicken the halo because that water will be reboiled virtually as quickly as it is imbibed. Thus, the thickness of the halo is not expected to grow significantly over the life of the dry zone. Because the halo is expected to remain thin, the volume of water in the halo is not expected to be sufficient to rewet the formation back to the repository level after radioactive decay has lowered the nuclear decay heat to low levels.

In this analysis it has been assumed that the fracture network is well connected and mobile water is not trapped anywhere in the network. If local regions exist that do not have a connected fracture network, those regions will pressurize from nuclear decay heating and may fracture. These individual fractures will connect with other parts of the formation, increasing the fracture connectivity through the formation. It is noted that a zone of low fracture permeability may inhibit fluid flow but still allow the vapor to escape to depressurize the system. 


\subsection{Conclusions}

As the dry zone expands around the repository, the temperature of water in the matrix will exceed its saturation temperature and vaporize. This vaporization will increase the pressure in the matrix and drive the water vapor into the fracture network and away from the repository. This water vapor will enter cooler regions of the formation and condense in the fractures. Because the fracture porosity is so low, a water bank cannot form above the repository unless a significant amount of water enters the matrix. The two primary transfer mechanisms for water to enter the matrix are a nonequilibrium capillary imbibition between the fracture and matrix and non-equilibrium hydrostatic pressures driving gravity drainage.

In this report, analytical expressions for the growth rate of the dry zone were developed. These expressions allow the flow rate of water entering the fracture network above the dry zone from condensation to be determined. Analytical expressions for the water transfer mechanisms of capillary imbibition and gravity drainage between the fracture and matrix were also developed. These expressions were then used to determine the characteristic water transport rates through the formation.

The velocity of water draining through the fractures by gravity drainage was found to be as much as six orders of magnitude faster than the velocity of the boiling front around the expanding dry zone. The velocity of water imbibing from the fractures into the matrix by capillary pressure was on the same order as the velocity of the boiling front. The velocity of water entering the matrix from the fractures by gravity drainage was several orders of magnitude lower than that from capillary imbibition. Thus, the rate that water drains away from the repository is many orders of magnitude higher than both the rate that water enters the fracture from the expanding dry zone and the rate that water leaves the fractures and enters the matrix.

The results of this study are consistent with the G-Tunnel test results, which indicated no water bank forming above the dry zone, and differ from the simulation results based on the equivalent continuum model (ECM), which indicated a water bank may form above the dry zone. The reason that the simulation studies predict the creation of a water bank is that the ECM assumes capillary equilibrium between the fractures and matrix. This study quantified the non-equilibrium fluid flow between the fractures and matrix and has shown that water entering the fractures above the dry zone will drain away from the repository before capillary equilibrium could be created. Thus, the ECM models are inappropriate for many studies of water transport around the proposed Yucca Mountain repository. It is noted that the authors of the ECM studies recognized this limitation and recommended that the more difficult non-equilibrium studies be conducted.

It is concluded that a significant water bank above the repository from the redistribution of water from nuclear decay heating is unlikely. Thus, the integrity of the repository is not expected to be threatened by rewetting of the formation from a water bank. 


\section{References}

Buscheck, T. A. and Nitao, J. J., "The Impact of Repository Heat on ThermoHydrological Performance at Yucca Mountain," Proceedings American Nuclear Society Topical Meeting on Site Characterization and Model Validation (Focus 93), Las Vegas, NV, Sept. 26-30, $1993 a$.

Buscheck, T. A. and Nitao, J. J., "Repository-Heat Driven Hydrothermal Flow at Yucca Mountain, Part I: Modeling and Analysis," Nuclear Technology, Vol. 104, pp. 418-448, Dec. $1993 b$.

Buscheck, T. A. and Nitao, J. J., "The Impact of Buoyant Gas-Phase Flow and Heterogeneity on Thermal-Hydrological Behavior at Yucca Mountain," International High Level Radioactive Waste Management Conference, American Nuclear Society, May 22-26, 1994.

Buscheck, T. A., Nitao, J. J., and Saterlie, S. F., "Evaluation of Thermo-Hydrological Performance in Support of the Thermal Loading Systems Study," Proceedings American Nuclear Society International High Level Radioactive Water Management Conference, Las Vegas, NV, May 22-26, 1994.

Buscheck, T. A., Nitao, J. J., and Wilder, D. G., "Repository-Heat Driven Hydrothermal Flow at Yucca Mountain, Part Il: Large-Scale In Situ Heater Tests," Nuclear Technology, Vol. 104, pp. 449-471, Dec. 1993.

Carslaw, H. S. and Jaeger, J. C., Conduction of Heat in Solids, Oxford University Press, New York, p.75, 1959.

Cil, M. and Reis, J. C., "A Multi-Dimensional, Analytical Model For Counter-Current Water Imbibition into Gas-Saturated Matrix Blocks," J. Pet. Sci. and Eng., in press, 1995.

Lin, W., private communication, Lawrence Livermore National Laboratory, 1994.

Nelson, R. A., Geologic Analysis of Naturally Fractured Reservoirs, Gulf Publishing Co., Houston, 1985.

Nitao, J. J., "Theory of Matrix and Fracture Flow Regimes in Unsaturated Fractured Porous Media," Proc. 2nd Annual International Conference on High Level Radioactive Waste Management, American Nuclear Society, Las Vegas, NV, April 28-May 3, 1991.

Nitao, J. J., "User's Manual for USNT Module of the NUFT Code, Version 1.0," Lawrence Livermore National Laboratory, 1993.

Nitao, J. J., "Reference Manual for the NUFT Flow and Transport Code, Version, 1.0," Lawrence Livermore National Laboratory, 1994. 
Nitao, J. J. and Buscheck, T. A., "Infiltration of a Liquid Front in an Unsaturated Fractured Porous Medium," Water Resources Research, Vol. 27, p. 2099, 1991.

Nitao, J. J., Buscheck, T. A., and Chesnut, D. A., "Implications of Episodic Nonequilibrium Fracture-Matrix Flow on Repository Performance," Nuclear Technology, Vol. 104, pp. 385-402, Dec. 1993.

Pirson, S. J., Boatman, E. M., and Nettle, R. L., "Prediction of Relative Permeability Characteristics of Intergranular Reservoir Rocks from Electrical Resistivity Measurements," J. Pet. Tech., pp. 564-570, 1964.

Reis, J. C., "An Analysis of Oil Expulsion Mechanisms From Matrix Blocks During Steam Injection in Naturally Fractured Reservoirs, In Situ, Vol. 16, No. 1, pp. 4373, 1992.

Roberts, J., private communication, Lawrence Livermore National Laboratory, 1994. 


\section{Nomenclature}

\section{English}

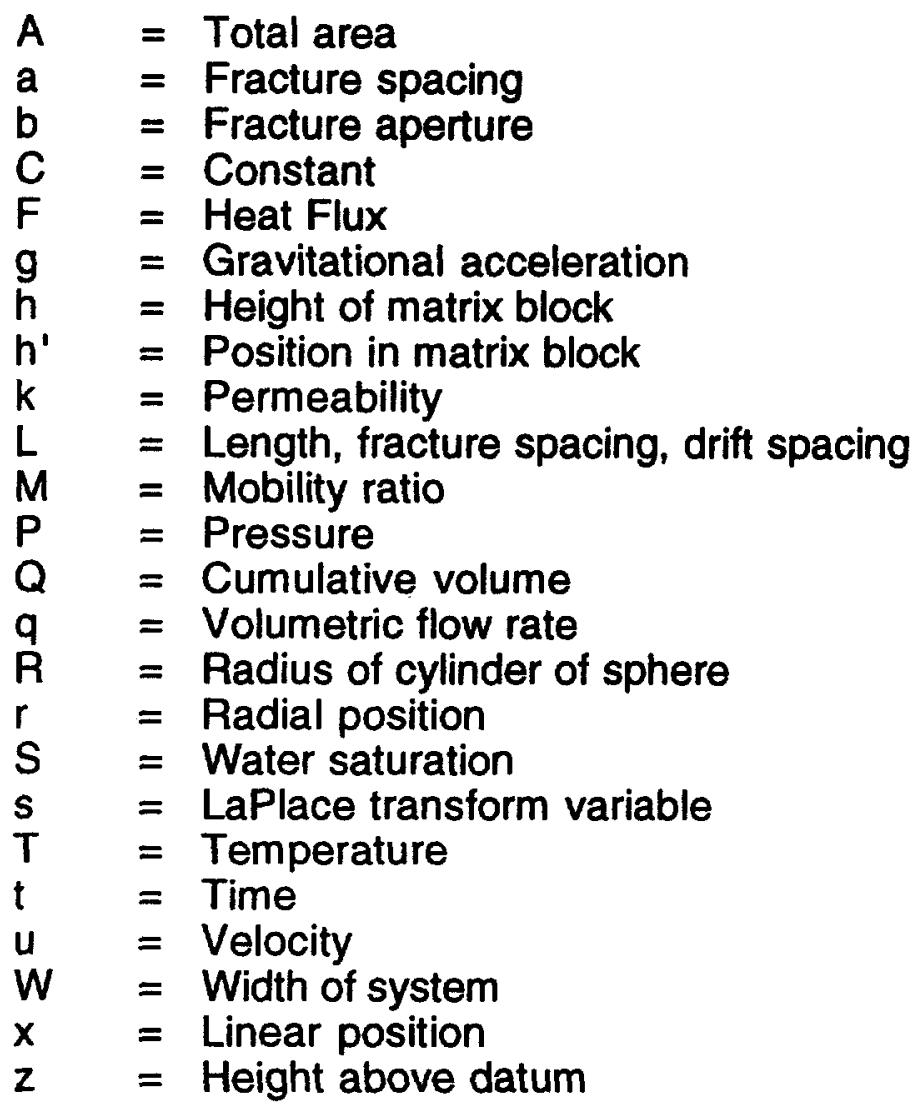

\section{Greek}

$$
\begin{array}{ll}
\alpha & =\text { Thermal diffisivity } \\
\beta & =\text { Imbibition parameter } \\
\Delta & =\text { Change } \\
\gamma & =\text { Local time since passage of front } \\
\phi & =\text { Porosity } \\
\Phi & =\text { Flow Potential } \\
\lambda & =\text { Thermal conductivity } \\
\mu & =\text { Viscosity } \\
\theta & =\text { Angle } \\
\rho & =\text { Density }
\end{array}
$$


Subscripts

$$
\begin{array}{ll}
\text { acc } & =\text { Accumulation rate } \\
\text { avg } & =\text { Average } \\
\text { b } & =\text { Bulk, bottom, boiling } \\
c & =\text { Capillary, characteristic } \\
D & =\text { Dimensionless } \\
d & =\text { Dry zone } \\
\text { f } & =\text { Fracture, front } \\
g & =\text { Gas } \\
h & =\text { Top of matrix block } \\
h^{\prime} & =\text { Position in matrix block } \\
\text { i } & =\text { Injection rate, initial } \\
\text { im } & =\text { Imbibition } \\
\text { L } & =\text { Local } \\
\text { lay } & =\text { Layer } \\
\text { loss } & =\text { Loss rate } \\
m & =\text { Matrix } \\
r & =\text { Radius } \\
\text { rg } & =\text { Relative to gas } \\
r w & =\text { Relative to water } \\
t & =\text { Top } \\
w & =\text { Water } \\
w b & =\text { Water bank } \\
w i & =\text { Water initial } \\
\text { wib } & =\text { Water bank, initial } \\
w m & =\text { Water maximum } \\
x & =\text { Position } \\
0 & =\text { Initial }
\end{array}
$$


J. C. Reis

August 1995

Table 2.1.1 Data for Temperature Models

\begin{tabular}{|l|l|l|}
\hline Parameter & Value & Units \\
\hline Initial temperature & 23 & ${ }^{\circ} \mathrm{C}$ \\
\hline Linear heat flux & 25 & $\mathrm{~W} / \mathrm{m}^{2}$ \\
\hline Cylindrical heat flux & 80 & $\mathrm{~W} / \mathrm{m}^{2}$ \\
\hline Spherical heat flux & 160 & $\mathrm{~W} / \mathrm{m}^{2}$ \\
\hline Thermal conductivity & 1.5 & $\mathrm{~W} / \mathrm{m} /{ }^{\circ} \mathrm{C}$ \\
\hline Thermal diffusivity & $2.44 \times 10^{-7}$ & $\mathrm{~m}^{2} / \mathrm{s}$ \\
\hline Time & 100 & years \\
\hline Boiling temperature & 96 & ${ }^{\circ} \mathrm{C}$ \\
\hline Radius of cylinder and sphere & 5 & $\mathrm{~m}$ \\
\hline Spacing between cylinders & 50 & $\mathrm{~m}$ \\
\hline Spacing between spheres & 50 by 20 & $\mathrm{~m}$ \\
\hline
\end{tabular}


J. C. Reis

August 1995

Table 2.2.1 Data for Numerical Simulations

\begin{tabular}{|l|l|l|}
\hline Parameter & Value & Units \\
\hline Porosity & 0.25 & fraction \\
\hline Initial water saturation & 0.4 & fraction \\
\hline Irreducible water saturation & 0.4 & fraction \\
\hline Water viscosity & 0.0005 & $\mathrm{~kg} / \mathrm{m} / \mathrm{s}$ \\
\hline Thermal diffusivity & $4.78 \times 10^{-07}$ & $\mathrm{~m}^{2} / \mathrm{s}$ \\
\hline Permeability & $9.869 \times 10^{-13}$ & $\mathrm{~m}^{2}$ \\
\hline Water density & 1000 & $\mathrm{~kg} / \mathrm{m}^{2}$ \\
\hline Gravitational acceleration & 9.81 & $\mathrm{~m} / \mathrm{s}^{2}$ \\
\hline Boiling temperature & 100 & ${ }^{\circ} \mathrm{C}$ \\
\hline Initial temperature & 15 & ${ }^{\circ} \mathrm{C}$ \\
\hline Thermal conductivity & 1.5 & $\mathrm{~W} / \mathrm{m} /{ }^{\circ} \mathrm{C}$ \\
\hline Heat source strength & 40 & $\mathrm{~W}$ \\
\hline
\end{tabular}


Table 2.2.2 Data for Matrix Pressurization

\begin{tabular}{|l|l|l|}
\hline Parameter & Value & Units \\
\hline Porosity & 0.11 & fraction \\
\hline Gas viscosity & 0.0137 & cp \\
\hline Effective permeability & 1 & microdarcy \\
\hline Average pressure & 12.6 & psi \\
\hline
\end{tabular}


J. C. Reis

August 1995

Table 3.1.1 Data for Water Bank Height

\begin{tabular}{|l|l|l|}
\hline Parameter & Value & Units \\
\hline Boiling Temperature & 96 & ${ }^{\circ} \mathrm{C}$ \\
\hline Initial Temperature & 23 & ${ }^{\circ} \mathrm{C}$ \\
\hline Heat flux & 25 & $\mathrm{~W} / \mathrm{m}^{2}$ \\
\hline Thermal conductivity & 1.5 & $\mathrm{~W} / \mathrm{m} /{ }^{\circ} \mathrm{C}$ \\
\hline Thermal diffusivity & $2.44 \mathrm{E}-07$ & $\mathrm{~m}^{2} / \mathrm{s}$ \\
\hline Capillary pressure & 165 & $\mathrm{psi}$ \\
\hline Gravitational acceleration & 9.81 & $\mathrm{~m} / \mathrm{s}^{2}$ \\
\hline Permeability & 1 & microdarcy \\
\hline Relative permeability, air & 0.1 & fraction \\
\hline Relative permeability, water & 0.1 & fraction \\
\hline Water viscosity & 0.001 & $\mathrm{~Pa}-\mathrm{s}$ \\
\hline Air viscosity & $9.68 \times 10^{-6}$ & $\mathrm{~Pa}-\mathrm{s}$ \\
\hline Porosity & 0.11 & fraction \\
\hline Saturation change during imbibition & 0.27 & fraction \\
\hline Saturation change during drying & 0.68 & fraction \\
\hline
\end{tabular}


J. C. Reis

August 1995

Table 3.2.1 Data for Gravity Drainage

\begin{tabular}{|l|l|l|}
\hline Parameter & Value & Units \\
\hline Bulk permeability & 280 & millidarcy \\
\hline Water density & 960 & $\mathrm{~kg} / \mathrm{m}^{3}$ \\
\hline Gravitational acceleration & 9.81 & $\mathrm{~m} / \mathrm{s}^{2}$ \\
\hline Matrix porosity & 0.11 & fraction \\
\hline Saturation change in matrix & 0.27 & fraction \\
\hline Viscosity of water & 0.3 & $\mathrm{cp}$ \\
\hline
\end{tabular}

* These permeabilities are the effective permeability, e.g., the product of the absolute and relative permeabilities. 
Table 3.3.1 Data for Merging Dry Zones

\begin{tabular}{|l|l|l|}
\hline Parameter & Value & Units \\
\hline Spacing between drifts & 38.4 & $\mathrm{~m}$ \\
\hline Porosity & 0.11 & fraction \\
\hline Initial water saturation & 0.68 & fraction \\
\hline Water density & 961 & $\mathrm{~kg} / \mathrm{m}^{3}$ \\
\hline Water latent heat & 2270 & $\mathrm{~kJ} / \mathrm{kg}$ \\
\hline Thermal diffusivity & $4.78 \times 10^{-07}$ & $\mathrm{~m} 2 / \mathrm{s}$ \\
\hline Thermal conductivity & 1.5 & $\mathrm{~W} / \mathrm{m} /{ }^{\circ} \mathrm{C}$ \\
\hline Boiling temperature & 96 & ${ }^{\circ} \mathrm{C}$ \\
\hline Initial formation temperature & 23 & ${ }^{\circ} \mathrm{C}$ \\
\hline Heat flux & 15 & $\mathrm{~W} / \mathrm{m}^{2}$ \\
\hline Distance along drift & 1 & $\mathrm{~m}$ \\
\hline
\end{tabular}


Table 3.4.1 Data for Imbibition

\begin{tabular}{|l|l|l|}
\hline Parameter & Value & Units \\
\hline Matrix block height & 4 & inches \\
\hline Permeability & 1 & microdarcy \\
\hline Relative permeability of water & 0.1 & fraction \\
\hline Relative permeability of air & 0.1 & fraction \\
\hline Viscosity of water & 1.0 & $\mathrm{cp}$ \\
\hline Viscosity of air & 0.0097 & $\mathrm{cp}$ \\
\hline Porosity & 0.099 & fraction \\
\hline Saturation change & 0.80 & fraction \\
\hline Water density & 1000 & $\mathrm{~kg} / \mathrm{m}^{3}$ \\
\hline Air density & 0.007 & $\mathrm{~kg} / \mathrm{m}^{3}$ \\
\hline Gravitational acceleration & 9.81 & $\mathrm{~m} / \mathrm{s}^{2}$ \\
\hline Capillary pressure & 165 & $\mathrm{psi}$ \\
\hline
\end{tabular}




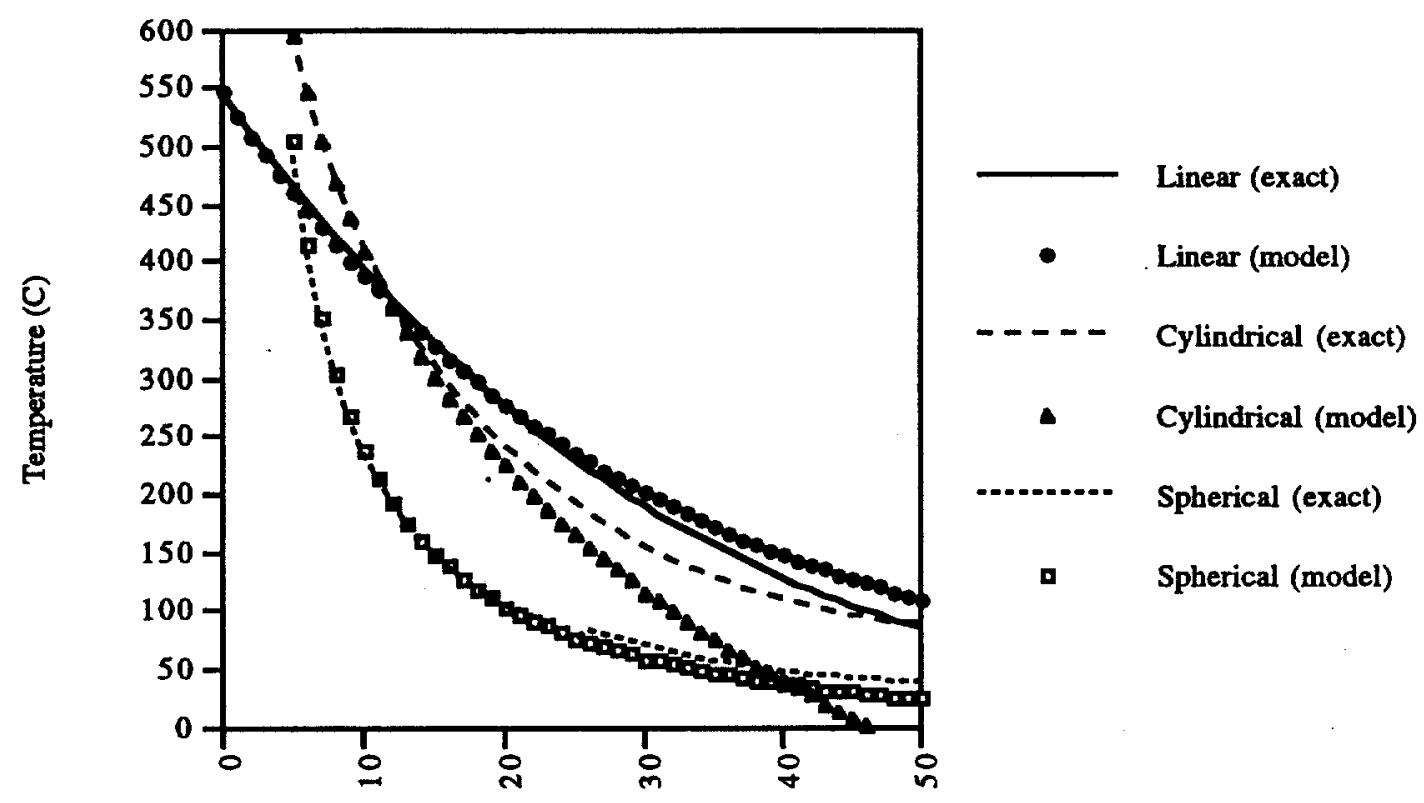

Position (m)

Figure 2.1.1 Comparison of Temperature Models for Conduction 


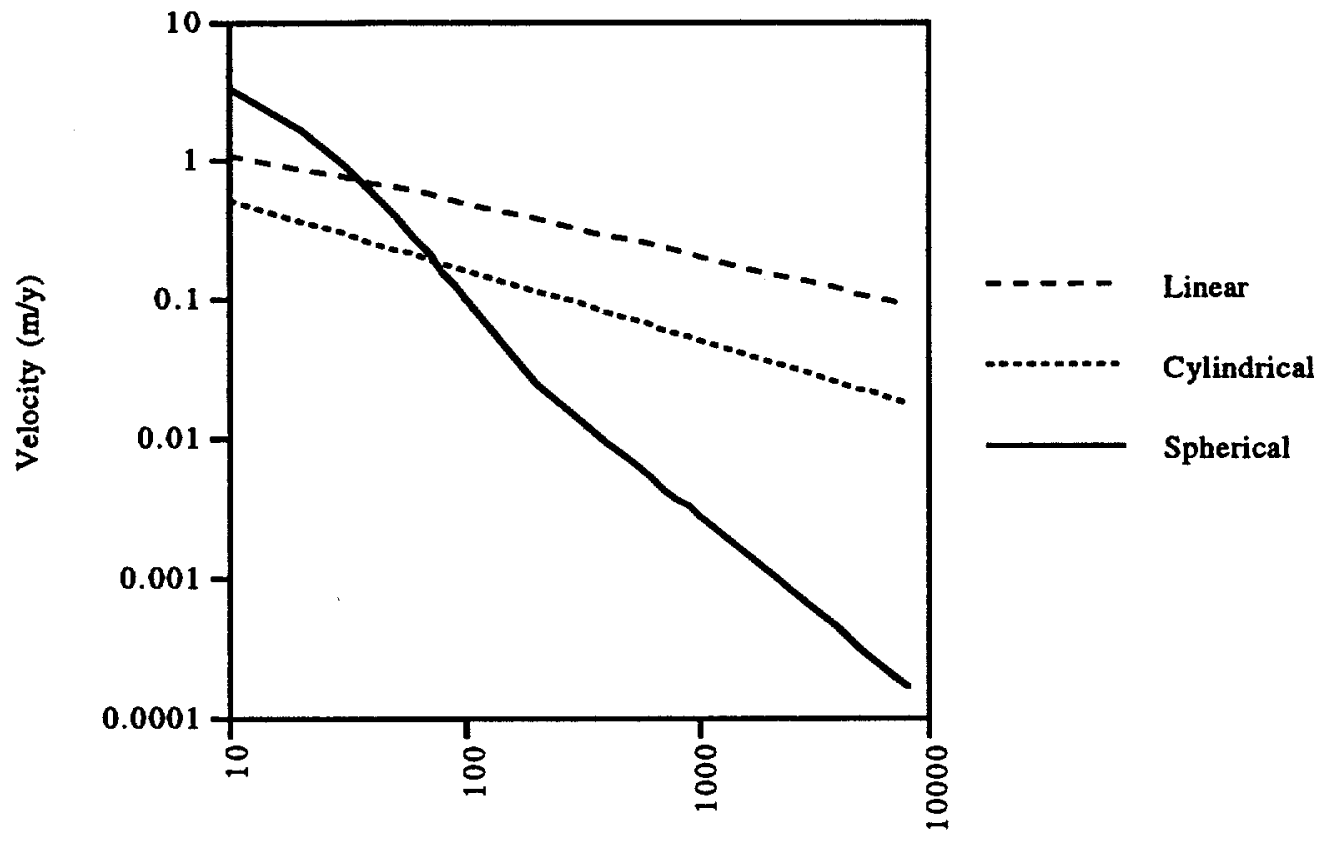

Time (years)

Figure 2.1.2 Boiling Front Velocities 


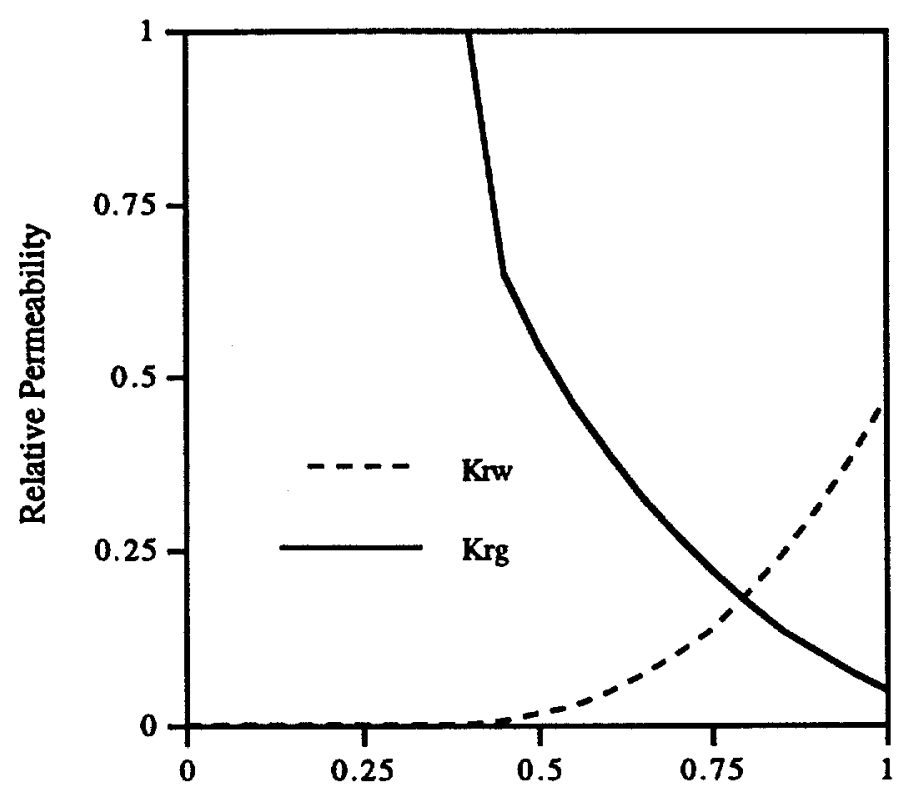

Water Saturation

Figure 2.1.3 Relative Permeabilities for Simulation Model 


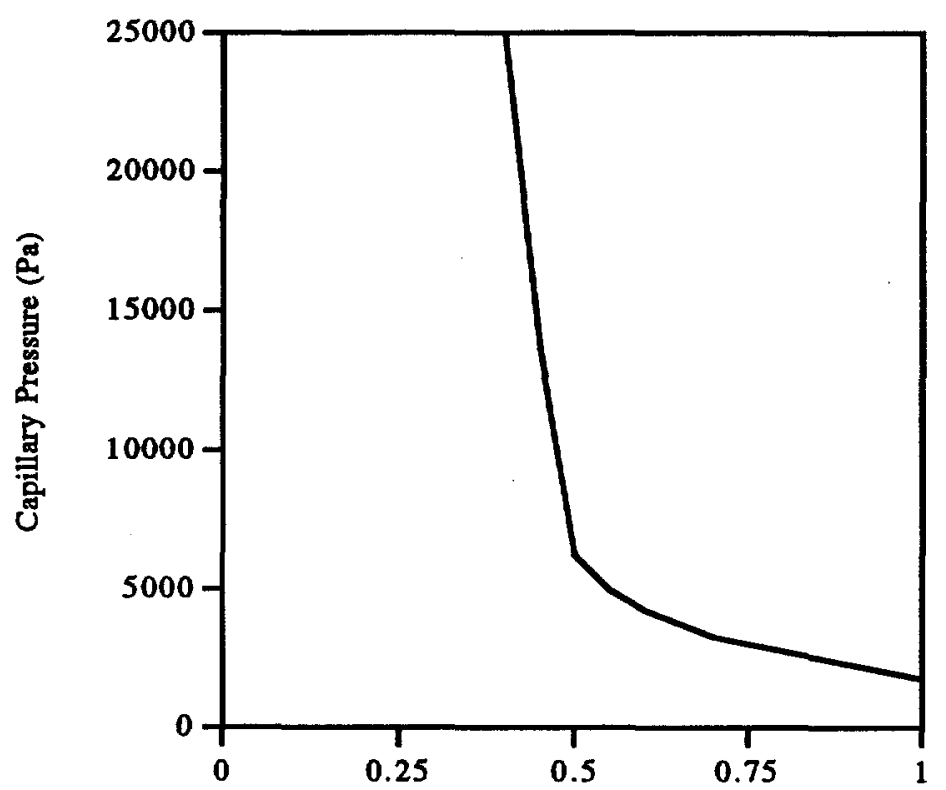

Water Saturation

Figure 2.1.4 Capillary Pressure for Simulation Model 
J. C. Reis

August 1995

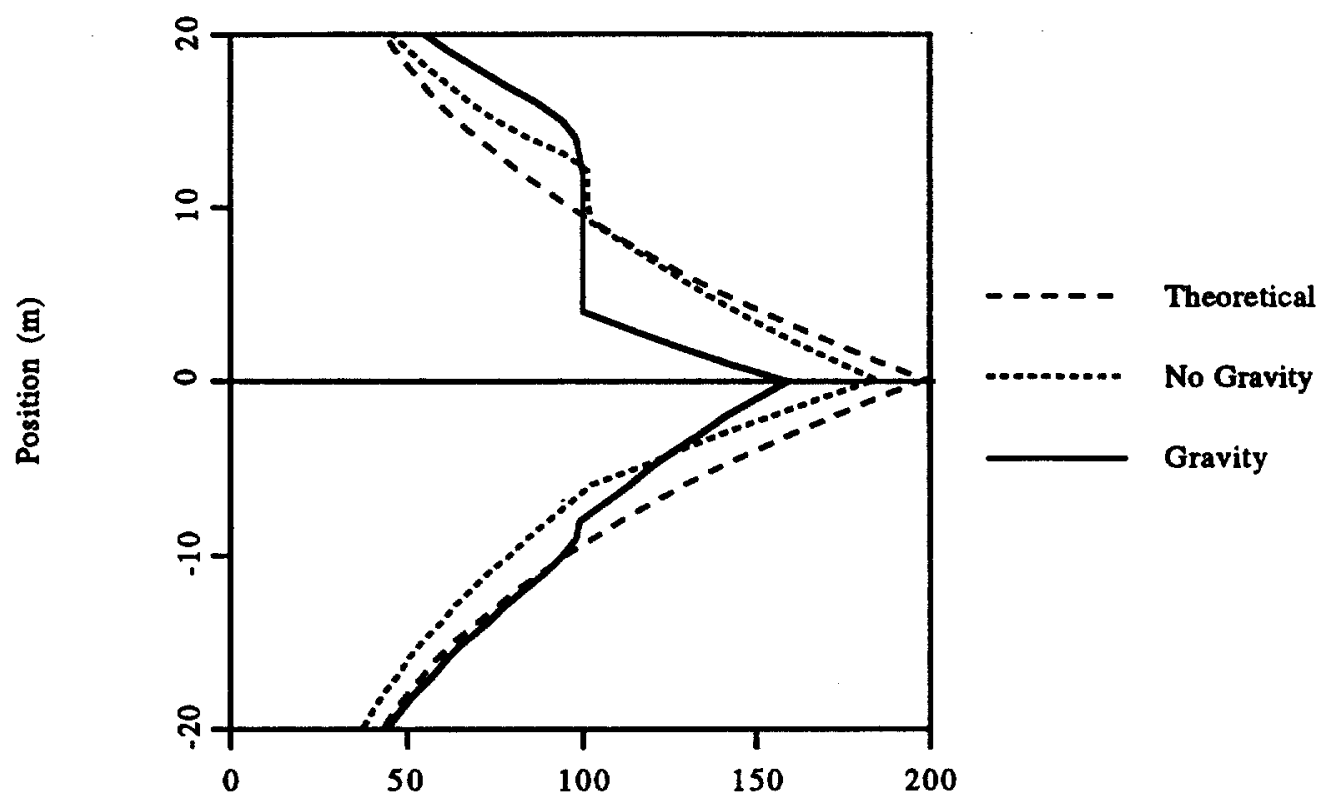

Temperature (C)

Figure 2.1.5 Temperature Profiles for Constant Pressure Boundary 


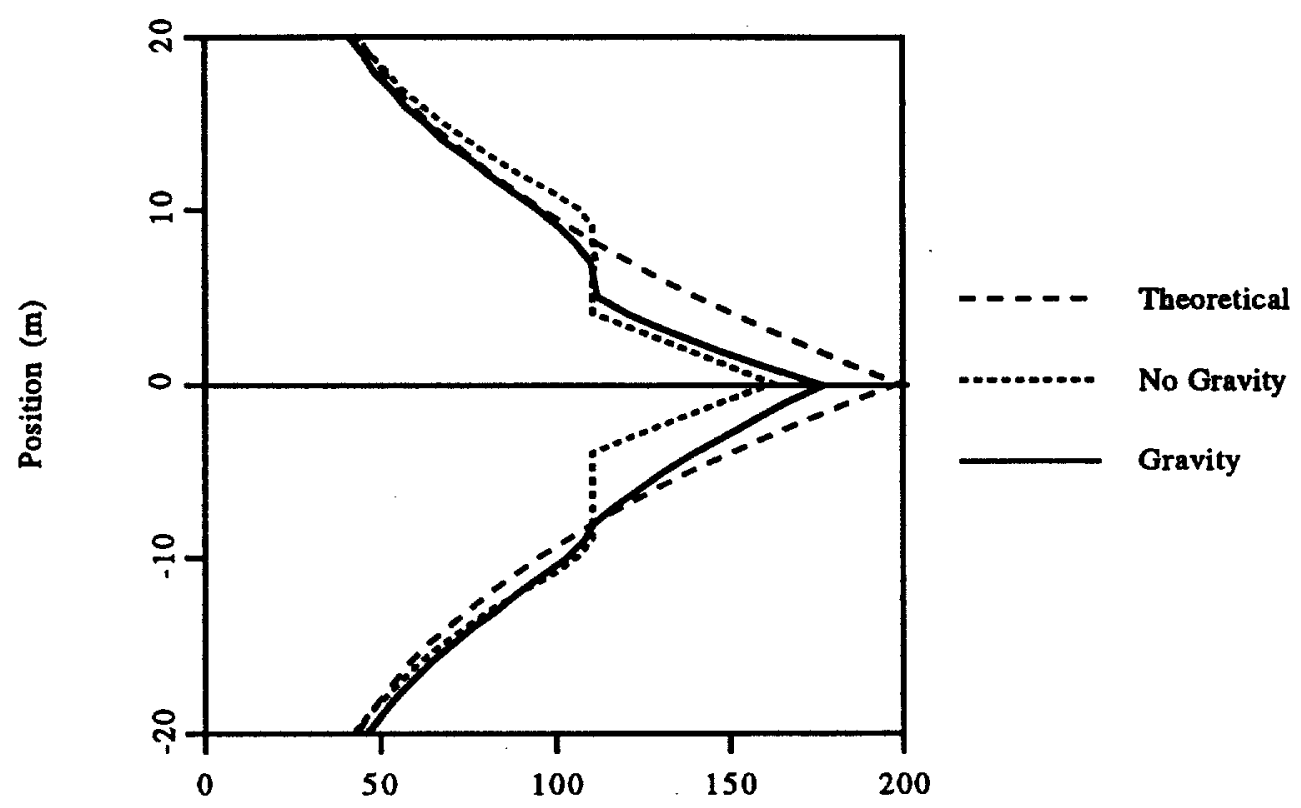

Temperature (C)

Figure 2.1.6 Temperature Profiles for No-Flow Boundary 


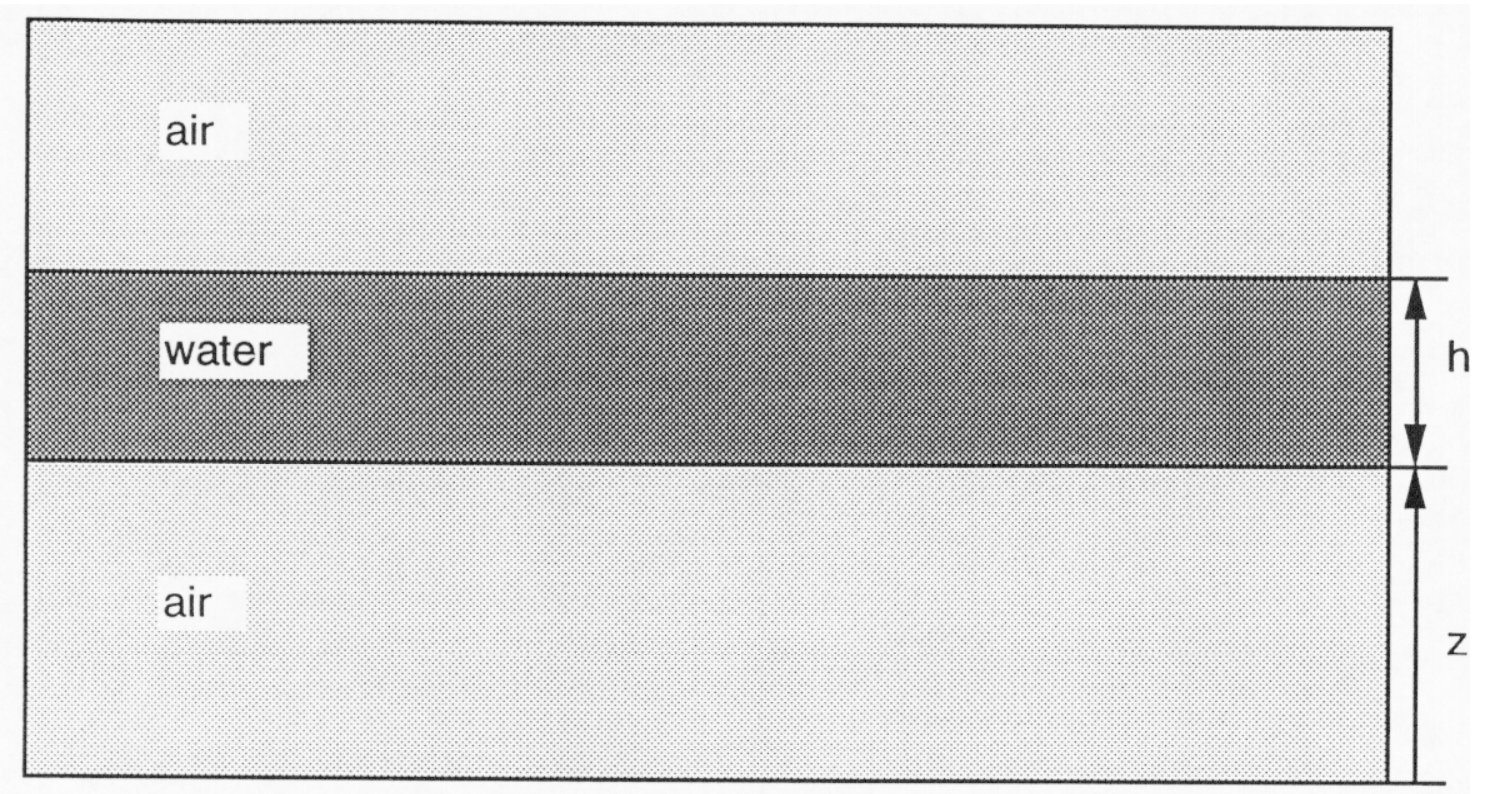

Figure 2.2.1 Schematic of Water Bank During Gravity Drainage 


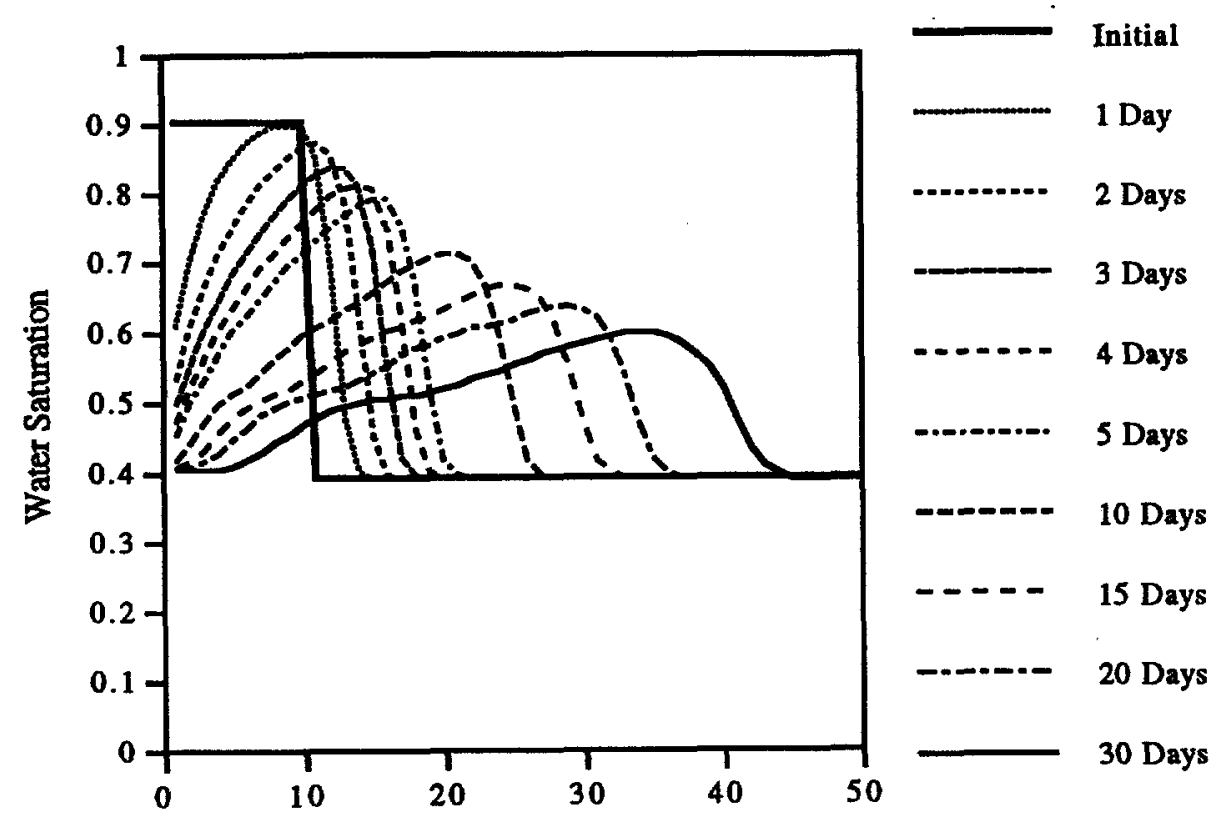

Distance (m)

Figure 2.2.2 Water Saturation Profiles During Gravity Drainage 


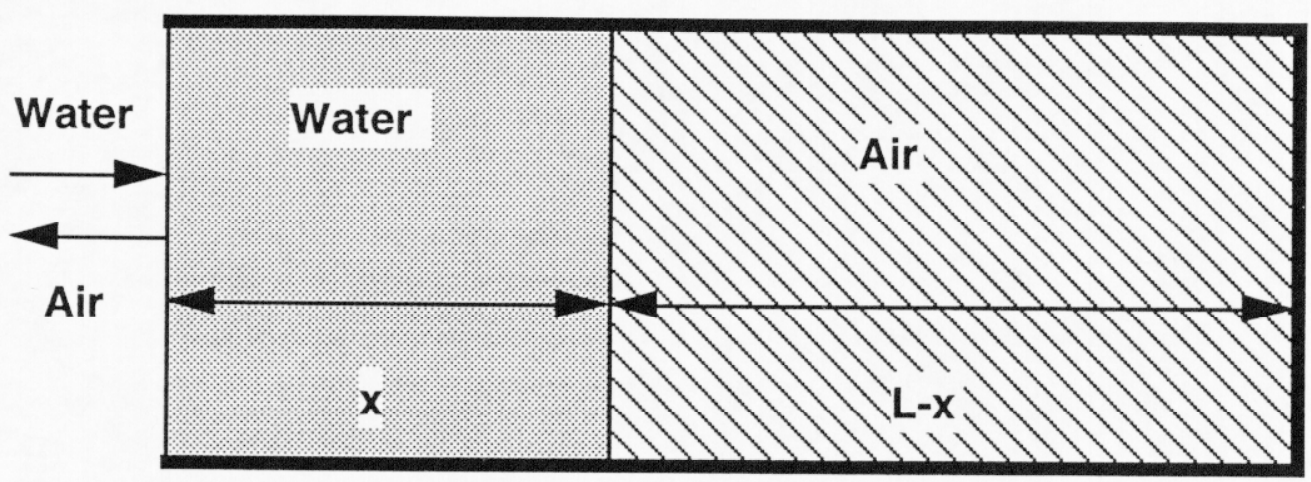

Figure 2.2.3 Geometry for Counter-Current Capillary Imbibition 


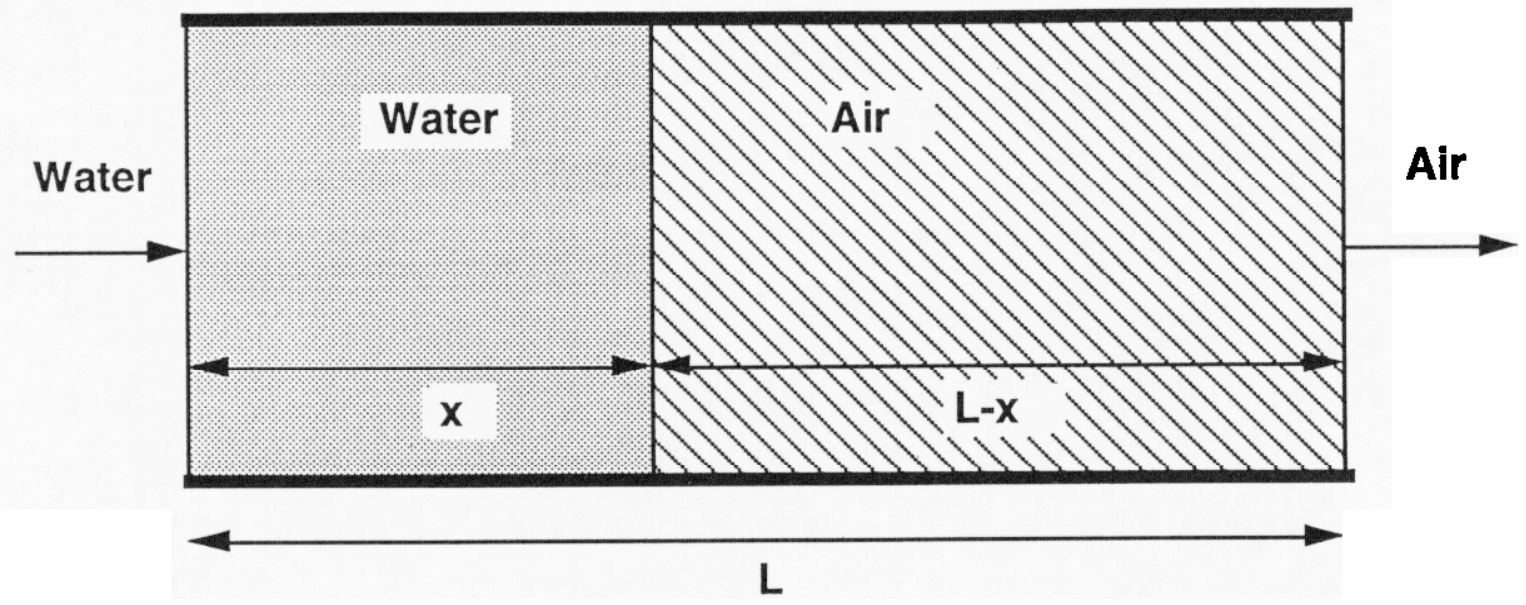

Figure 2.2.4 Geometry for Co-Current Capillary Imbibition 
J. C. Reis

August 1995

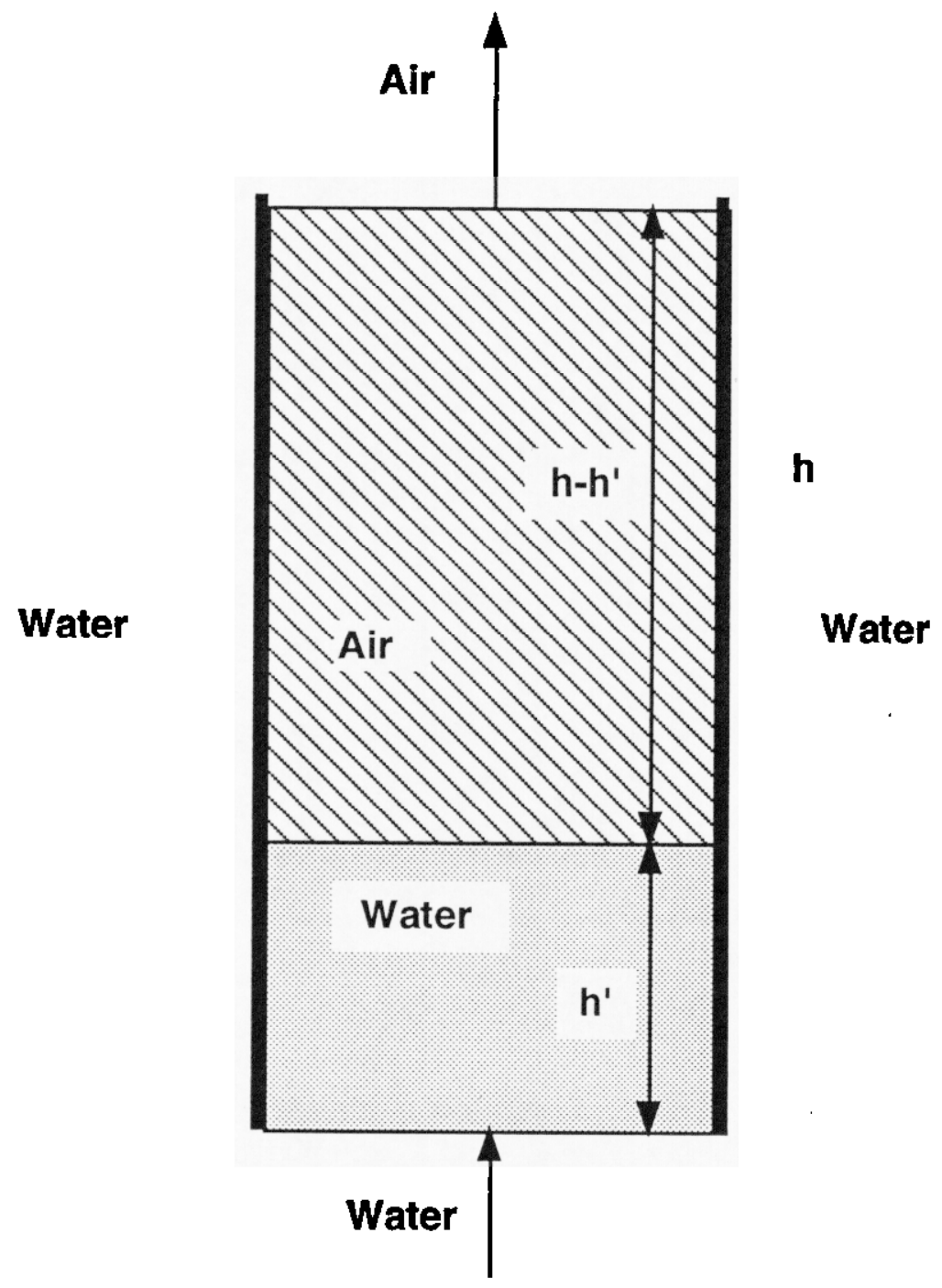

Figure 2.2.5 Geometry for Co-Current Capillary and Gravity Imbibition 


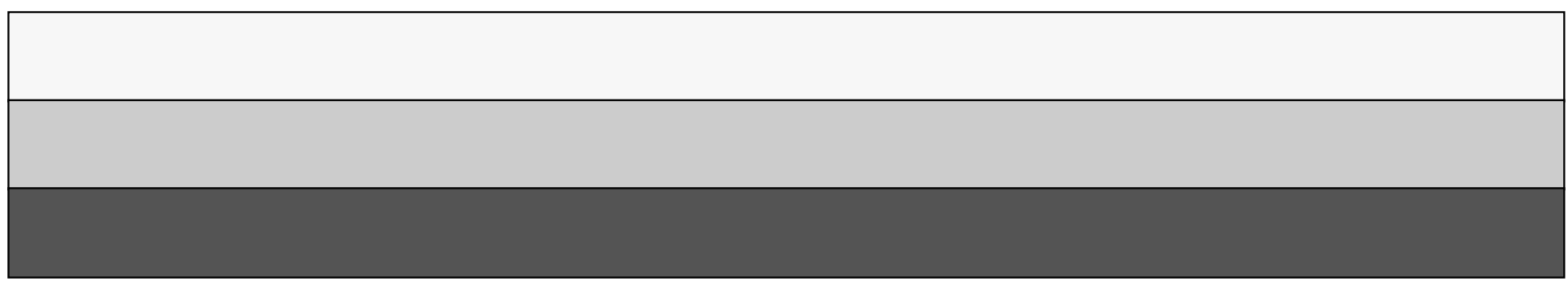

